\title{
CD20 as a gatekeeper of the resting stage of human B cells
}

Kathrin Kläsener ${ }^{1,2}$, Julia Jellusova ${ }^{1,2}$, Geoffroy Andrieux ${ }^{3,4}$, Ulrich Salzer ${ }^{5}$, Chiara Böhler ${ }^{1,2}$, Sebastian N. Steiner6,7, Jonas B. Albinus ${ }^{6,7}$, Marco Cavallari ${ }^{1,2}$, Beatrix Süß ${ }^{8}$, Melanie Boerries $^{3,4,9}$, Reinhard E. Voll ${ }^{5}$, Bernd Wollscheid ${ }^{6,7}$, and Michael Reth ${ }^{1,2 *}$

1 Biology III, faculty of biology, University Freiburg

2 Research Centres BIOSS and CIBSS

3 Institute of Medical Bioinformatics and Systems Medicine, Medical Center - University of Freiburg, Faculty of Medicine, University of Freiburg, Freiburg 79110, Germany

4 German Cancer Consortium (DKTK) Partner Site Freiburg, German Cancer Research Center (DKFZ), Heidelberg, Germany

5 Department of Rheumatology and Clinical Immunology, Medical Center - University of Freiburg, Faculty of Medicine, University of Freiburg, Freiburg, Germany

6 Institute of Translational Medicine at the Department of Health Sciences and Technology, ETH Zurich, 8093 Zurich, Switzerland.

7 Swiss Institute of Bioinformatics (SIB), Lausanne, Switzerland.

8 Department of Biology, Centre for Synthetic Biology, Technical University Darmstadt, Darmstadt, Germany

9 Comprehensive Cancer Center Freiburg (CCCF), Medical Center-University of Freiburg, University of Freiburg 79106, Germany

\section{*For correspondence:}

Michael Reth

E-mail: michael.reth@bioss.uni-freiburg.de

Phone: +49 761 203-2718

Biology III, Faculty of Biology, University Freiburg

Schänzlestr. 1 D-79104 Freiburg

\section{Abstract}

$\mathrm{CD} 20$ is a B cell specific membrane protein and a target of therapeutic antibodies such as rituximab $(\mathrm{RTX})^{1}$. In spite of the prominent usage of anti-CD20 antibodies in the clinic little is known about the biological function of CD202. Here we show that $\mathrm{CD} 20$ controls the nanoscale organization of receptors on the surface of resting B lymphocytes. A CRISPR/Cas-based ablation of CD20 in Ramos B cells results in a relocalisation of the IgM B cell antigen receptor (IgM-BCR) and the co-receptor CD19. The resulting IgM-BCR/CD19 signaling synapse leads to transient B cell activation followed by plasma cell differentiation. Similarly to CD20deficient Ramos cells, naïve human B cells treated with rituximab in vitro or isolated from patients during rituximab administration display hallmarks of transient activation characterized by the formation of the IgM-BCR/CD19 signaling synapse, followed by CD19 and IgM-BCR downregulation. Moreover, increased expression of specific plasma cell genes can be observed after rituximab treatment in relapsed CLL patients. In summary we identify CD20 as a gatekeeper of the resting state on human B cells and demonstrate that a disruption of the 
nanoscale organization of the $\mathrm{B}$ cell surface via CD20 deletion or anti-CD20 treatment profoundly alters B cell fate.

The human Burkitt lymphoma cell line Ramos carries large amounts of the IgM-BCR on its cell surface, whereas the IgD-BCR is less abundant on these cells (Fig 1a). Ramos B cells also express the co-receptor CD19 and the B cell-specific membrane protein CD203-5. The B cellspecific expression of CD20 makes it an ideal target for therapeutic antibodies used for the treatment of human B cell neoplasias and autoimmune diseases such as rheumatoid arthritis or systemic lupus erythematosus ${ }^{6-8}$. We have previously localized the CD19/CD81 coreceptor module together with CD20 and CD40 inside an IgD-class specific membrane compartment on the surface of resting $\mathrm{B}$ cells ${ }^{9-11}$. To determine the nanoscale organization of the CD20 molecule on the Ramos B cell surface, we used the Fab-based proximity ligation assay (Fab-PLA). Similar to our previous studies on primary B cells, we find CD20 in close proximity to the IgDBCR on resting Ramos cells whereas after a pervanadate-mediated B cell activation CD20 moves to the IgM-BCR (Fig 1b). Thus, the nanoscale membrane organization of CD20 seems to be similar on Ramos and primary B cells (Fig 1c).

Using the CRISPR/Cas9 technology we targeted exon 3 of the MS4A1 gene encoding CD20 and disrupted the open reading frame of this gene shortly after the ATG start codon (Extended Data Figure 1). The obtained CD20KO Ramos cell lines were classified according to the date of gene targeting as new (KO-N), intermediate (KO-I), and late (KO-L) cells (Fig 2a). The KOI and KO-L cells were negatively selected for the loss of CD20 expression by cell sorting. In total we generated 18 distinct CD20KO Ramos cell lines (Supplementary Table 5) that were analysed at different time points for the abundance of selected cell surface markers. Surprisingly, the KO-I and KO-L Ramos cells not only lost the surface expression of CD20, but also that of other B cell surface markers such as CD19, CD22, CD81, CD40 and the IgMBCR (Fig 2b). The altered protein abundance was confirmed by Western blotting analysis of lysates from KO-L cells which showed a loss, or marked reduction of CD20, CD19, CD22, CD40, IgM and CD81 proteins in comparison to Ramos wild type (WT) cells (Fig 2c). Thus, the CRISPR/Cas9-induced CD20 gene deficiency is accompanied by drastic changes of the receptor abundances on the surface of Ramos cells and the loss of the B cell resting stage. 
Similarly to KO-I and KO-L cells, KO-N Ramos cells show reduced expression of CD20, CD22, CD81 and CD40, however CD19 and IgM-BCR levels are increased (Fig 2b). On resting murine and human B cells, CD19 and the IgM-BCR are separated from each other but form a signaling synapse upon B cell activation ${ }^{9,12}$. Interestingly, an increased IgM:CD19 proximity is detected by Fab-PLA on KO-N Ramos cells, indicating that these cells are activated (Fig $2 \mathbf{d}$ ). Indeed, a phosphoflow cytometry analysis of WT and KO-N Ramos cells revealed an increased phosphorylation of Syk and Akt in the KO-N population with the highest IgM expression (Fig 2e). Furthermore, KO-N cells upregulate the B cell activation markers CD86 and CD69 (Fig 2f). Thus, the CD20 gene deletion is associated with a transient B cell activation followed by a loss/downregulation of several B cell surface markers. Together, these data suggest that on resting $\mathrm{B}$ cells CD20 functions as a gatekeeper preventing uncontrolled IgM-BCR/CD19 interaction and signaling.

To learn more about the molecular requirements for the changes of B cell surface expression displayed by the CD20KO Ramos cells we generated CD20 loss mutants from Ramos CD19KO and from BCR-KO cells, deficient in heavy chain, light chain and activation induced deaminase (AID) expression. Interestingly, none of the double KO (DKO) Ramos cells displayed the transient B cell activation (data not shown). Unlike KO-L, the CD19/20DKO-L Ramos cells still express the IgM-BCR and CD22 (Extended Data Figure 2a), whereas the BCR/20DKOL Ramos cells retain expression of the B cell surface marker CD19 and CD22 (Extended Data Figure 2b). Together this analysis suggests that the BCR and CD19 interaction is indispensable for the transient B cell activation and the altered surface expression of CD20KO Ramos B cells.

\section{Re-expression of CD20 restores the resting $B$ cell phenotype}

We next asked whether the re-expression of CD20 could restore the expression of cell surface proteins on CD20-deficient Ramos B cells. For this we established a conditional KO (cKO) of the CD20 encoding MS4A1 gene (Extended Data Figure 3). Using the CRISPR/Cas9 technique, we inserted between exon 3 and exon 4 of the MS4A1 gene, an aptamer-controlled conditional exon (c-exon) whose incorporation in the processed MS4A1 mRNA results in a stop code thus rendering this transcript defective ${ }^{13}$. Upon exposure of the Ramos cKO cells to tetracycline (Tet) the antibiotic molecule binds to and stabilizes an aptamer whose sequence partly overlaps with the 3' splice site of the c-exon thus preventing its incorporation into the CD20 transcript (Extended Data Figure 3a). This synthetic tetracycline riboswitch enables the successful regulation of CD20 protein levels. Similar to KO-L, the cKO Ramos B cells cultured 
112 for more than 30 days do not express CD20 nor CD19, IgM-BCR, CD22, CD81 or CD40 on

113 the B cell surface (Extended Data Figure 3b). An overnight (o/n) Tet-exposure of the cKO

114 Ramos cells restores the expression of CD20 and that of the other B cell markers on the cell

115 surface. Thus, upon its re-expression, CD20 can resume its gatekeeper function to support the

116 resting state of Ramos B cells.

118 Rituximab treatment of naïve human B cells terminates the gatekeeper function of CD20

119 CD20 is a prominent target of therapeutic antibodies such as rituximab (RTX) that are used for 120 the depletion of B lymphocytes in autoimmune diseases and B cell neoplasias. To test whether 121 the RTX treatment interferes with the gatekeeper function of CD20 we used flow cytometry to 122 monitor the expression of B cell surface markers on WT Ramos cells (Fig 3a), and healthy 123 donor (HD) derived naïve B cells (Fig 3b) before or after a RTX treatment. Interestingly, all 124 RTX-treated cells lost CD20 from the surface and simultaneously downregulated CD19, CD22 125 and the IgM-BCR to a similar extent as the KO-L Ramos cells. To test for the immediate effect 126 of RTX in vivo we analysed blood samples taken from patients suffering from rheumatoid 127 arthritis (RA) undergoing RTX treatment. B cells from these patients showed a transient 128 increase of surface IgM-BCR expression already after $15 \mathrm{~min}$, followed by a continuous IgM129 BCR downregulation. CD19 surface expression, which is routinely used as a B cell marker was 130 almost lost after 30 min of RTX treatment, although B cells were still present and detectable 131 (Fig 3c). A Fab-PLA study showed an increased BCR-IgM:CD19, Iga:pSyk, Ig $\alpha: p Y p 110 \delta$ and 132 Ig $\alpha:$ pAkt proximity in RTX-treated compared to untreated HD B cells, indicating that the RTX 133 treatment is accompanied by increases of BCR-associated Syk/PI-3kinase signaling and B cell 134 activation (Extended Data Figure 4a and 4b). In summary, these data show that CD20 is a 135 gatekeeper also for resting naïve human B cells and that a CD20 deficiency or RTX treatment 136 terminates B cell dormancy.

\section{Dynamic alteration of the surfaceome of CD20-deficient B cells}

139 To obtain a more global picture of the alteration of protein expression on the surface of CD20140 deficient Ramos B cells we took advantage of the Cell Surface Capture (CSC) technology ${ }^{14,15}$.

141 CSC technology allows for the efficient labelling, identification and relative quantification of $142 \mathrm{~N}$-glycosylated proteins residing on the cell surface and provides a snapshot of the surfaceome 143 without having to use antibodies to broadly phenotype the B cells ${ }^{16}$. The CSC analysis of the 144 3-7-day- old KO-N Ramos cells shows a significant upregulation of surface markers (3 down 145 versus 45 up, p-value $<0.05$ ) that is consistent with the activated state of these cells (Extended 
146 Data Figure 5a and 5b). The proteins with higher cell surface abundance include the IgM-

147 BCR and CD19, thereby confirming our previous flow cytometry analysis (Fig. 2b).

148 Interestingly, the loss of CD20 on KO-N Ramos cells resulted in the upregulation of several

149 tetraspanins such as CD37, CD53, CD63, CD82, and TSN3 that could contribute to nanoscale

150 membrane organization as well ${ }^{17}$. In contrast to KO-N, the KO-L Ramos cells display a

151 reduction in the cell surface abundance of many proteins (123 down versus 58 up, p- value

$152<0.05)$, indicating that at a later time point the CD20 deficiency is associated with gene

153 silencing (Extended Data Figure 5c and 5d). Indeed, the abundance of many B cell markers 154 such as the BCR, CD19, CD38, CD27 and CD22 is reduced on KO-L Ramos cells.

155 Interestingly, several surface proteins upregulated on KO-L Ramos cells are also found to be 156 highly abundant on the surface of plasma cells. This suggests that the loss of CD20 is not only 157 associated with a loss of the resting B cell stage but also an increased differentiation towards 158 the plasma cell stage.

\section{Plasma cell differentiation of CD20-deficient B cells}

161 In culture, with time CD20KO Ramos cells become larger as indicated by the increased forward scatter (FSC) and they upregulate TACI and CD138, both plasma cell markers. (Fig 4a). An intracellular FACS analysis showed that CD20KO Ramos cells upregulate AID at the early KO-N stage where these cells display active Syk/PI-3 kinase signaling. Thus, similar to activated $\mathrm{B}$ cells participating in a germinal center $(\mathrm{GC})$ reaction the $\mathrm{CD} 20 \mathrm{KO}$ Ramos seem to undergo somatic hypermutation and class switching. Indeed, the KO-L Ramos cells carry IgA on their cell surface and secrete IgA (Fig 4b). In normal activated B cells, the differentiation to the plasma cell stage is associated with a transcriptional switch involving the down-regulation of the transcription factor PAX5 and upregulation of Blimp-1. Western blot analysis shows that this is also the case in CD20KO Ramos cells (Fig 4c). In comparison to WT, the KO-L Ramos cells express less PAX5 and more Blimp-1. Furthermore, KO-L Ramos cells contain more

172 FoxO1, IRF4 and XBP1s, transcription factors that are associated with $\mathrm{B}$ to plasma cell 173 differentiation. Another known regulator of plasma cell differentiation is the Blimp-1 suppressor and transcriptional silencer Bcl6, that is highly expressed in GC B cells as well as GC-derived B lymphomas such as Ramos ${ }^{18-21}$. Indeed, Bcl6 is expressed in WT Ramos but no

176 longer detectable in the KO-L cells. The PAX5 to Blimp-1 transcriptional switch was not 177 detected in the CD19/20DKO-L and BCR/20DKO-L Ramos cells, indicating that BCR/CD19 178 signaling and transient $\mathrm{B}$ cell activation was required for this in-vitro human plasma cell 179 differentiation (Extended Data Figure 6c and 6d). We next asked whether a RTX treatment 
is also accompanied by an increased plasma cell differentiation of B cells in vivo. For this we analysed the transcriptome profile of B cells from CLL patients ${ }^{22}$ that have relapsed after a combined RTX treatment (RTX \pm Fludarabine) for the expression of plasma cell specific genes in a gene set enrichment analysis (GSEA) and compared this gene set with Ramos WT and CD20KO-L cells (Fig 4e). Interestingly, the samples from 11 of 13 CLL-patients showed a significantly increased expression of plasma cell-specific genes after combined RTX-treatment, confirming our results that CD20-derived B cell activation can induce activation of the plasma cell transcription program (Fig $\mathbf{4} \mathbf{f}$ and $\mathbf{4 g}$ ).

\section{Distinct alterations of cell metabolism during Ramos plasma cell development}

Plasma cells are large cells with an extended endoplasmic reticulum (ER) and a high rate of antibody production. Plasma cells thus have a higher demand for energy and biosynthetic precursor molecules than B lymphocytes ${ }^{23,24}$. To characterize the metabolic program of WT and KO-L Ramos cells we performed metabolic flux experiments and untargeted metabolomics analyses. We found the abundance of 117 biochemicals to be significantly increased and the abundance of 214 biochemicals to be significantly decreased in KO-L Ramos cells in comparison to WT cells. Analysis of the oxygen consumption rate (OCR) showed that KO-L cells consume more oxygen in the basal state and possess a higher spare respiratory capacity than WT Ramos cells (Fig 5a).

199 Nicotine amid adenine dinucleotide (NAD+) is a crucial cofactor for enzymes in several

200 metabolic pathways and high levels of NAD+ have been suggested to favor mitochondrial 201 biogenesis and function ${ }^{25}$. We found mitochondrial mass (Fig 5b) and NAD+ levels (Fig 5c) to be significantly increased in KO-L Ramos cells in comparison to WT cells. Since reactive oxygen species (ROS) are a natural by-product of respiratory activity, we hypothesized that ROS production might be altered in KO-L Ramos cells. Indeed, we found $\mathrm{O}_{2}{ }^{-}$levels to be increased (Fig 5d) in KO-L Ramos cells. Moreover, we found the glutathione-cysteine ligase inhibitor buthionine sulfoximine (BSO) to induce cell death of KO-L Ramos cells, and less so in WT cells (Fig 5e). Glutathione-Cysteine Ligase is an important component of the

208 Glutathione-dependent ROS scavenging pathway and protects cells from ROS induced cellular damage. Our results thus suggest that KO-L Ramos cells are exposed to increased levels of ROS and that adaptations to oxidative stress are crucial for their survival.

211 We next compared the glycolytic activity of WT and KO-L Ramos cells by measuring the 212 extracellular acidification rate (ECAR). As expected for a Burkitt lymphoma, WT Ramos cells 213 showed robust increase of ECAR after receiving glucose (Fig 5f). Upon oligomycin treatment, 
which is an inhibitor of mitochondrial ATPase, ECAR was further increased to compensate for the loss of mitochondria-derived ATP. In contrast to WT cells, KO-L cells showed low lactate production upon receiving glucose, yet ECAR levels were similar to those seen in WT after oligomycin treatment (Fig 5f). This suggests that although KO-L cells are capable of converting glucose to lactate this metabolic pathway is not favored under normal conditions. Consistent with their reduced glycolytic rates, the KO-L cells showed reduced expression of hexokinase II (HKII), the enzyme mediating the first step of glycolysis (Fig 5g). Furthermore, we found the levels of glycolytic intermediates such as glucose 6-phosphate, fructose 6-phosphate, and pyruvate to be decreased in KO-L cells (Fig 5h). Taken together these data suggest that glucose is not the primary source of energy in KO-L Ramos cells.

To assess whether alternative carbon sources play a more prominent role in KO-L Ramos cells, we treated the cells with Bis-2-(5-phenylacetamido-1,3,4-thiadiazol-2-yl)-ethyl sulphide (BPTES), an inhibitor of glutaminolysis. We found that while BPTES treatment induced cell death of KO-L cells, WT Ramos cells were less affected (Fig 5i). Furthermore, unlike WT Ramos, KO-L cells can be cultured in a medium where glucose is replaced by galactose (Fig 5k). KO-L cells are able to process galactose intracellularly and use it as an alternative carbon source (Fig 5l). Unlike glucose, galactose needs to be catabolized in the mitochondria to provide cells with energy. Thus, our results demonstrate that KO-L Ramos cells can survive entirely without glycolysis-derived ATP.

233 In summary, our metabolic analysis demonstrates that the loss of CD20 results in profound 234 metabolic alterations which in many instances mirror the phenotype of plasma cells/plasma 235 blasts $^{26,27}$.

\section{Discussion}

Macfarlane Burnet's clonal selection theory provided the foundation for the explanation of adaptive immunity. The proposed selection process requires a strict separation between resting and activated B lymphocytes. We show here that CD20 is a gatekeeper of the resting B cell state. On resting mature B lymphocytes, the IgM-BCR and the IgD-BCR reside on the cell surface inside separated nanodomains with distinct lipid and protein compositions. Together with CD19, CD20 is a component of the IgD-class nanodomains. Upon antigen-dependent B cell activation, this nanoscale receptor organization is altered and CD19 together with the IgMBCR forms a signaling synapse. We find that the exposure to anti-CD20 antibodies in vitro and

246 the loss of CD20 also results in IgM/CD19 synapse formation, association to Syk/PI-3 kinase

247 signaling and transient B cell activation by itself. 
248 CD20 deficiency in mice and humans has been associated with reduced B cell signaling and T

249 cell-independent immune responses rather than increased BCR signaling ${ }^{28,29}$. These differences 250 may be due to compensation mechanism operating during $\mathrm{B}$ cell selection ${ }^{30,31}$. With the 251 CRISPR/Cas9 technique it is feasible to establish a CD20 deficiency at the mature B cell stage 252 without interference of the B cell selection process and to combine several gene $\mathrm{KO}$ for a gene 253 function analysis. In this way, we could show that the transient B cell activation is associated 254 with plasma cell differentiation of all our Ramos cell lines. With an unbiased GSE 255 transcriptome analysis we found plasma cell-specific genes upregulated after combined RTX 256 treatment in the transcriptome of about $85 \%$ of the investigated patients ${ }^{22}$. Disruption of the 257 nanoscale organization of CD20 followed by development of plasma cells could explain the 258 results of a study on immune thrombocytopenia patients after RTX medication which suggests 259 that B cell depletion generates a milieu that promotes the differentiation and settlement of long260 lived PC in the spleen ${ }^{32,33}$.

262 It is known that plasma cells improve metabolic flexibility ${ }^{34,35}$ to secure function and prolonged 263 survival. Our analysis of the metabolic program of KO-L Ramos cells allowed us to identify 264 metabolic vulnerabilities in these cells. Our finding that CD20KO Ramos cells switch from 265 glycolysis to oxidative phosphorylation and from glucose to glutamine usage is in line with 266 previous studies of plasma cell metabolism ${ }^{24,25}$. Importantly, the insight obtained from this 267 metabolic profiling enabled us to identify BPTES and BSO as inhibitors reducing the viability 268 of KO-L Ramos cells and these compounds provide a rationale to target these metabolic 269 pathways in plasma cell pathologies. To find plasma cell characteristics and adaptations on 270 CD20 negative cancer B cells make a successful treatment more challenging but enables new 271 possibilities for patients that have relapsed after RTX medication. They may benefit from a 272 treatment tailored to make use of the specific biological properties of plasma cells. 


\section{Material and Methods}

\section{Primary human naïve B cells}

276 Primary naive B cells were obtained from fresh buffy coats provided by the Institute for

277 Transfusion Medicine and Gene Therapy, ITG Freiburg. Peripheral blood mononuclear cells 278 (PBMCs) were separated by Ficoll gradient centrifugation of whole blood and negatively 279 selected using EasySep Human Naïve B Cell Isolation kit (StemCell). Prior to the experiments, 280 primary naïve B cells were controlled for purity and rested overnight in complete RPMI 1640 281 supplemented with 5\% FCS, 10 units/ml penicillin/streptomycin (Gibco), 20 mM HEPES 282 (Gibco), and $50 \mathrm{mM} \beta$-mercaptoethanol (Sigma) at $37^{\circ} \mathrm{C}$ with $5 \% \mathrm{CO}_{2}$. Experiments with 283 primary human B cells were repeated at least three times. This study was approved by the 284 Institutional Review Board of the University Freiburg (Ethical votes No 507/16 and 336/16).

\section{Cell culture}

287 The human Burkitt lymphoma B cell-line Ramos was obtained from ATCC, Ramos (RA 1) 288 (ATCC ${ }^{\circledR}$ CRL-1596 ${ }^{\mathrm{TM}}$ ). Ramos cells were cultured in RPMI 1640 medium (Gibco), supplemented 289 with 5\% FCS (Biochrom), 10 units/mL penicillin/streptomycin (Gibco), 20 mM HEPES 290 (Gibco), and $50 \mathrm{mM} \beta$-mercaptoethanol (Sigma) at $37^{\circ} \mathrm{C}$ with $5 \% \mathrm{CO}_{2}$. For glucose versus 291 galactose metabolism analysis, Ramos B cells were cultured in RPMI 1640 medium without 292 glucose (Gibco), supplemented with $11 \mathrm{mM}$ sterile filtered glucose or galactose, respectively, $2935 \%$ dialyzed FCS (Thermo Fisher), 10 units / $\mathrm{mL}$ penicillin / streptomycin (Gibco), $20 \mathrm{mM}$ 294 HEPES (Gibco), and $50 \mathrm{mM} \beta$-mercaptoethanol (Sigma). Cells were cultured at $37^{\circ} \mathrm{C}$ with 5\% $295 \mathrm{CO}_{2}$.

\section{CRISPR/Cas9 knock out}

298 CRISPR/Cas9 KO plasmids used in this study are listed in Supplementary Table 1. 299 CRISPR/Cas9 deletion was either carried out using the Neon Transfection System (Invitrogen) 300 to either deliver the Human MS4A1 KO plasmids (Santa Cruz) into the cells or the 301 ribonucleoprotein according to the genome editing method of Integrated DNA Technology $302(\text { IDT })^{36}$.

303 For plasmid delivery, $1.1 \times 10^{6}$ cells were resuspended with $110 \mu \mathrm{L}$ transfection medium 304 containing $20 \mathrm{mM}$ HEPES (Gibco) and 1.25\% DMSO (Sigma) in RPMI medium and then 305 mixed together with $4 \mu \mathrm{g}$ of KO plasmid. The cells were then subjected to Neon Transfection 306 System. Electroporation was performed in $100 \mu \mathrm{L}$ NEON tips at $1350 \mathrm{~V}, 30 \mathrm{~ms}$ and a single 
pulse and recovered at $37^{\circ} \mathrm{C}$ and $5 \% \mathrm{CO}_{2}$ without antibiotics for one day and then cultured in complete RPMI-medium.

309 For the IDT CRISPR-Cas9 approach equimolar amounts of Alt-R CRISPR-Cas9 crRNA and tracrRNA were annealed in IDTE buffer, combined with Cas9 endonuclease before complex

311 formation with RNP. One million cells were then subjected to Neon Transfection System.

312 Electroporation was performed in $10 \mu \mathrm{L} \mathrm{NEON} \mathrm{tips} \mathrm{at} 1350 \mathrm{~V}$, with a single $30 \mathrm{~ms}$ pulse. The 313 transfected cells were cultured at $37^{\circ} \mathrm{C}$ and $5 \% \mathrm{CO}_{2}$ in complete RPMI medium. Inactivation 314 of the target gene was verified by flow cytometry and/or western blotting.

\section{Flow cytometry analysis}

317 For surface staining, 1-20 × $10^{5}$ cells were stained with antibodies in PBS supplemented with $3180.5 \%$ BSA and $0.05 \% \mathrm{NaN}_{3}$ on ice for $20 \mathrm{~min}$, washed twice and then measured with a FACS 319 Gallios (Beckman Coulter) For intracellular staining, the cells were fixed with 4\% PFA, washed 320 twice in PBS and permeabilized with $0.5 \%$ BSA containing $0.5 \%$ saponine for 60 min at room 321 temperature. Data were exported in FCS-3.0 format and analyzed with FlowJo software 322 (TreeStar). A BIO-RAD S3e cell sorter was used to select CD20-negative cell populations.

323 Flow cytometry analysis were performed more than three times from each of the eighteen 324 independently generated CD20KO Ramos B cell lines. Detailed information about primary 325 antibodies are listed in Supplementary Table 2.

\section{Lymphocyte and B-cell subpopulations phenotyping}

328 Phenotyping of T-, B- and NK cells within the lymphocyte population was performed by a whole blood staining lyse-no-wash protocol (Optilyse B, Beckman-Coulter) using six colour 330 flow cytometry with fluorochrome-conjugated antibodies as listed in Supplementary Table 7 331 top. Analysis of B-cell subpopulations was performed with a bulk lysis specimen of EDTA 332 anticoagulated whole blood treated with ammonium chloride lysis buffer to lyse erythrocytes. 333 After washing, a lyse-wash protocol (Optilyse B, Beckman-Coulter) was performed. To 334 determine B-cell subpopulations, nine colour flow cytometry with fluorochrome-conjugated 335 antibodies as listed in Supplementary Table 7 bottom was performed. Gating strategy in 336 Extended Data Figure 4b. Antibody labelled cells were analyzed by flow cytometry (Navios; 337 Beckman Coulter). Flow cytometric data analysis was performed with the help of Kaluza 338 Software 1.5a (Beckman Coulter).

\section{Cell survival}


Cell survival was determined by analyzing forward/sideward scatter properties of cells and simultaneous staining with either Propidium iodide (PI), or 7-amino-Actinomycin D (7AAD eFluor670 dye, and LIVE/DEAD fixable violet stain (ThermoFisher), following the manufacturer's instructions. Cell survival was continuously monitored in all experiments.

\section{Cell proliferation assay}

347 The cell proliferation assay was performed using CellTrace Proliferation Kit (ThermoFisher) 348 according to the manufacturer's protocol. In brief, one million cells were washed with PBS and stained in a $1 / 1000$ dilution of the stock solution for $20 \mathrm{~min}$ at $37^{\circ} \mathrm{C}$ protected from light. Cells were washed twice with complete supplemented RPMI. After 10 min the mean fluorescence intensity (MFI) was measured. Further readings were followed daily. MFI values were used to calculate the proliferation index ${ }^{37}$. Proliferation was measured at least three times.

\section{Western Blotting}

355 Cells were collected and immediately lysed in 2x Laemmli or RIPA buffer. Equal amounts of cleared lysates were subjected to SDS-PAGE on $10 \%, 12 \%$ or gradient (10-15\%) mini precast gels (7bioscience) and to subsequent immunoblotting on PVDF membrane (GE Healthcare Amersham) and blocked with 5\% BSA in PBS and 0.1\% Tween20. A horseradish (HRP) peroxidase- conjugated goat anti-rabbit or goat anti-mouse antibody was used as secondary antibody before detection with ECL chemiluminescent substrate (Bio-Rad). Western blot experiments were repeated more than three times. Lysates were taken from independently generated Ramos CD20KO B cell lines. Details of primary antibodies are shown in Supplementary Table 3.

\section{CRISPR riboswitch design and cKO Ramos cell generation}

366 The CRISPR riboswitch was designed based on a published exon-skipping RNA device ${ }^{13}$. A 367 minimized portion of the original, synthetic construct including part of the $5^{\prime}$ intron, the 3' 368 splice site, a tetracycline aptamer, the poised exon, the 5' splice site, and part of the 3' intron 369 was targeted to intronic sequences of the human CD20 gene. The tetracycline aptamers C1 and $370 \mathrm{C} 2$ used here correspond to those reported in the M1 and M2 minigene constructs ${ }^{13}$. The intronic 371 CRISPR guides were designed to avoid regulatory elements using the UCSC Genome Browser. 372 The Alt- ${ }^{\circledR}$ Cas9 ribonucleoprotein complexes were assembled as mentioned above ${ }^{36}$. The 373 riboswitch was amplified by PCR (CloneAmp, Takara; according to the manufacturer's 374 instructions) verified by agarose gel, and cleaned using a PCR purification kit. Details of 
primers used for the ex34 or ex45 homology directed repair (HDR) are listed in Supplementary

Table 1. The HDR templates were mixed at fourfold molar excess with a single-strand DNA binding protein (ET SSB, NEB, M2401S) and heated at $95^{\circ} \mathrm{C}$ for 10 minutes to achieve singlestranded donor oligonucleotides (ssODNs) bound to ET SSB. About 2 pmoles of HDR template were added to the RNP-enhancer-cell mixture and subjected to Neon Transfection System. Electroporation was performed in $10 \mu \mathrm{L}$ NEON tips at $1450 \mathrm{~V}$ with a single $20 \mathrm{~ms}$ pulse. For analysis four independent cKO Ramos B cells lines were created and Tet-induction experiments were performed at least four times.

\section{Surfaceome analysis using Cell Surface capture (CSC)}

For surfaceome screening 50 million cells per replicate of Ramos WT, CD20KO-N and CD20KO-L were harvested and washed with ice-cold PBS. CSC was performed as previously described $^{23}$. Deamidated peptides derived from N-glycosylated cell surface-residing receptors were analysed on a Q Exactive plus HF mass spectrometer (QE-HF MS) Thermo Scientific) coupled to an EASY-nLC 1200 instrument. The QE-HF MS was operated in positive ion mode and peptides were separated by reverse-phase chromatography on a $15 \mathrm{~cm}$ column in-house packed with ReproSil-Pur 120A C18-AQ $1.9 \mu \mathrm{m}$ (Dr. Maisch GmbH), primed with 100\% buffer $\mathrm{A}\left(99 \% \mathrm{H}_{2} \mathrm{O}, 01 \%\right.$ formic acid) and with a 70-minute gradient from 6 to $44 \%$ buffer B (99.9\% acetonitrile, $0.1 \%$ formic acid) prior to injection. Mass spectrometry data were acquired in data-dependent acquisition (DDA) mode (TOP10). MS1 scans were acquired with 60,000 resolution, AGC target of $3 \times 10^{6}$, IT of $15 \mathrm{~ms}$ and followed by high-energy collision dissociation (HCD) at 28\%. MS2 scans were recorded with 15,000 resolution, AGC target of $1 \times 10^{5}$ and IT of $110 \mathrm{~ms}$. Raw files were analysed using the Trans-Proteomic Pipeline (v4.6.2) by searching with COMET (v27.0) against Uniprot KB (Swiss-Prot, Homo sapiens, retrieved April 2018). Carbamidomethylation was set as a fixed modification for cysteine, oxidation of methionine and deamidation of arginine were set as variable modifications. Peptide feature intensities of identified peptides with FDR $\leq 1 \%$, presence of consensus NXS/T sequence and deamidation (+0.98 Da) at asparagines were extracted with Progenesis QI (v4.0, Nonlinear Dynamics) for label-free quantification. Statistical analysis was performed with MSstats (v 3.8.6) using iRT peptides to normalize across runs. Significance of differentially regulated proteins was determined by the threshold $\mid$ fold-change $\mid>1.5$ and $\mathrm{p}$-value $<0.05$ of a two-sided t-test with the appropriate degrees of freedom. Benjamini-Hochberg method was used to account for multiple testing. Protein abundance changes and significance was visualized in 
(https://carrotsearch.com/foamtree/). Hierarchical classification was created based on the 410 functional annotation of human plasma membrane proteins by Almén et al. ${ }^{38}$. CSC experiments 411 were performed in triplicates per condition. Acquired raw data will be deposited to PRIDE.

412 MS experiment details and link are shown in Supplementary Table 6.

413 The data (ID:PXD019874) will be public on PRIDE, as soon as the paper is accepted.

414 For review use the link: http://massive.ucsd.edu/ProteoSAFe/QueryPXD?id=PXD019874

415 Username: MSV000085605 Password: KathrinKlaesener

416 Once the data are public the link will be: ftp://massive.ucsd.edu/MSV000085605

\section{Proximity ligation assay (PLA)}

419 For Fab-PLA, the PLA-probes were prepared as previously described ${ }^{39}$. In brief, $420 \mathrm{~F}(\mathrm{ab})$ - fragments were prepared from the corresponding antibodies using the Pierce Fab Micro 421 Preparation Kit (Thermo Fisher) according to the manufacturer's protocol. In brief, after buffer 422 exchange (Zeba ${ }^{\mathrm{TM}}$ spin desalting columns, Thermo Fisher) F(ab)-fragments were coupled with 423 PLA probemaker Plus or Minus oligonucleotides according to the manufacturer's protocol 424 (Sigma-Aldrich) to generate Fab-PLA probes. For in situ PLA experiments, the cells were 425 allowed to attach to polytetrafluoroethylene (PTFE)-coated slides (ThermoFisher) for $30 \mathrm{~min}$ 426 at $37^{\circ} \mathrm{C}$. Depending on the experiment, the cells were activated with $1 \mathrm{mM}$ freshly prepared 427 pervanadate or treated with RTX and then fixed for 20 min with 4\% paraformaldehyde in PBS. 428 PLA was performed as described earlier ${ }^{39}$. PLA experiments were repeated at least three times, each in two technical replicates. Details about primary antibodies used for PLA-probes are provided in Supplementary Table 4.

\section{Imaging, Image analysis, and Data processing}

433 All microscope images were acquired using Leica DMi8 microscope equipped with a $63 \times$ oil 434 immersion objective lens. For each sample, several images of at least 1000 cells were imaged 435 from randomly chosen regions. All recorded images were analysed with CellProfiler 3.0.0. Raw 436 data produced by CellProfiler were exported to Prism software (GraphPad, La Jolla, CA). The mean PLA signal count per cell was calculated from the corresponding images and presented as scatter dot plots with mean and standard deviation (SD).

\section{Statistical analysis}

441 Nonparametric Mann-Whitney U-test was used for all experiments shown except results 442 obtained from the metabolome analysis. Statistical analysis of our metabolomics data was 
performed by Metabolon Inc and Welch's two samples t-test was used to determine statistical significance.

\section{ELISA of IgA-Secretion}

447 Quantification of total IgA antibody secretion was measured by sandwich ELISA (Thermo 448 scientific) with minor modifications. In brief, after centrifugation media aliquots of three serial 449 dilutions $(\mathrm{n}=3)$ of culture supernatants of KO-L and WT Ramos cells were added to 96-well 450 plates coated with $10 \mu \mathrm{g} / \mathrm{mL}$ of human anti lambda specific antibody and kept $\mathrm{o} / \mathrm{n}$ at $4^{\circ} \mathrm{C}$ to 451 calculate maximal effective concentration. RPMI and pure PBS served as a control. After extensive washes with wash buffer containing PBS, supplemented with $0.05 \%$ Tween20 and $0.01 \mathrm{NaN}_{3}$, non-specific binding was blocked using PBS, supplemented with 1\% BSA and $0.01 \% \mathrm{NaN}_{3}$. Binding was revealed using biotinylated anti-IgA secondary antibody followed by Streptavadin-Horseradish Peroxidase, developed with 3,3',5,5'-tetramethylbenzidine (TMB), and detected with Elisa microplate reader before normalization. Independently generated Ramos CD20KO-L cell lines were chosen for three repetitions. Details in

\section{Supplementary Table 4.}

\section{Inhibitors}

461 For inhibition Ramos cells were treated with Bis-2-(5-phenylacetamido-1,3,4-thiadiazol-2462 yl)ethyl sulfide (BPTES, final concentration of $0.5 \mu \mathrm{M}$ ) or L-Buthionine Sulfoximine (BSO, 463 final concentration $0-1600 \mu \mathrm{M}$, all Selleck-Chemicals). Inhibitors were diluted in RPMI 464 culture medium and treatment was performed as described in manufacturer's protocol.

\section{Rituximab (RTX) treatment}

RTX was kindly provided by F. Hoffmann-La Roche-AG. For Ramos cell treatment RTX was diluted to a final concentration of $10 \mu \mathrm{g} / \mathrm{mL}$ and performed at least three times.

\section{Transcriptome analysis}

471 Massive Analyses of cDNA Ends (MACE-Seq) was performed by GenXPro GmbH in 472 Frankfurt am Main using the MACE-Seq kit according to the manual of the manufacturers ${ }^{40}$. 473 Briefly, cDNA was generated with barcoded poly-A primers during reverse transcription. The 474 cDNA was fragmented and a second adapter was ligated. Competitive amplification was used 475 to produce a library that was sequenced on an Illumina NextSeq500 machine. The reads were 476 cleaned from adapter-residues and homopolymer stretches and annotated to the human genome 
(HG19) and read counts per gene was quantified. Differential gene expression was determined using the "DE-Seq2" package for p-value- and FDR calculation. (Technical replicates of Ramos WT $n=3$, biological replicates of independently generated CD20KO cell lines: KO-I n=4, and $\mathrm{KO}-\mathrm{L}, \mathrm{n}=5$ )

Differentially regulated genes between the different groups were identified using a linear model-based approach (limma R package ${ }^{41}$ ). Briefly genes with non-null read count in at least 2 samples were selected. After applying TMM normalization, a regular fitting method with unpaired design was used. Finally, genes with missing entrez IDs were filtered out for further analysis. Ajdusted p-value $<0.05$ was considered as significant.

\section{GEO data}

Microarray gene expression raw-data from 13 CLL patients treated with rituximab ${ }^{22}$ were downloaded from GEO (GSE37168). CEL files from samples before treatment and after relapse were RMA normalized using the oligo R package. Enrichment of plasma cell differentiation up-regulated genes was performed using the fgsea $\mathrm{R}$ package ${ }^{42}$. Genes were ranked according to their Log FC and used as input in fgsea. Genes for the individual patients were ranked according to the Log2 FC comparing the expression at relapse against before treatment from GSE37168. Plasma up-regulated genes are highlighted by the ticks on x-axis in fgsea, where color code represents the enrichment score during the random walk over the ranked list of genes. Statistical significance was assessed using 1000 permutations where p-value $<0.05$ was considered as significant. Plasma cell specific gene set is listed in Supplementary Table 8.

\section{Detection of mitochondrial mass}

501 To stain for mitochondrial mass, $10^{5}$ cells were stained in $100 \mu \mathrm{L} 60 \mathrm{nM}$ Mito Tracker502 RedCMXRos (Thermo Fisher) in Ramos medium for $30 \mathrm{~min}$ at $37^{\circ} \mathrm{C}$. Cells were washed twice 503 before measurement. Cells were analysed using a flow cytometer Gallios (Beckman Coulter).

504 Data were normalized according to cell volume. Three independent experiments have been 505 performed.

\section{Detection of mitochondrial ROS}

508 Detection of superoxide in mitochondria was performed with MitoSOX-Red mitochondrial 509 superoxide indicator (molecular probes) according to the manufacturer's protocol. In brief, $10^{5}$ 510 Ramos cells were loaded with $1 \mathrm{~mL}$ of reagent working solution $(5 \mu \mathrm{M}$ MitoSOX in HBSS- 
buffer) and incubated in the dark for $10 \mathrm{~min}$ in at $37^{\circ} \mathrm{C}$. The cells were washed three times with warm buffer and immediately subjected to imaging. Three replicate measurements were taken.

\section{Metabolomic analysis}

515 For metabolomic profiling, cells were washed with PBS and snap frozen in liquid nitrogen.

516 Samples were processed and analyzed by Metabolon Inc. The extracts were divided into five

517 fractions: two for analysis by two separate reverse phase (RP)/UPLC-MS/MS methods with 518 positive ion mode electrospray ionization (ESI), one for analysis by RP/UPLC-MS/MS with 519 negative ion mode ESI, one for analysis by Hydrophylic interaction chromatography 520 (HILIC)/UPLC-MS/MS with negative ion mode ESI, and one sample was reserved for backup.

521 Raw data was extracted and compounds were peak identified by Metabolon Inc. Missing values 522 were imputed with the observed minimum after normalization. Global metabolic profiles were 523 determined using UPLC-MS/MS (Metabolon). Shown are cell count normalized results for $524 \mathrm{NAD}^{+}$, G6P, F1.6BP, pyruvate, UDP-Galactose, Gal-1-P, Galactonate. The raw data obtained 525 from our metabolome studies will be deposited at MetaboLights upon manuscript acceptance.

\section{Metabolomic flux analysis}

528 To measure glycolytic flux, cells were resuspended in 50 $\mu 1$ Seahorse XF Base Medium 529 (Agilent) supplemented with 2mM L-glutamine (Thermo Fisher) and incubated for 30min at $53037^{\circ} \mathrm{C}$ in a $\mathrm{CO}_{2}$-free incubator. Subsequently, $130 \mu \mathrm{L}$ medium were added and cells were 531 incubated for 1h. ECAR was measured using the Seahorse XFe96 metabolic flux analyzer 532 (Agilent). Cells were sequentially treated with $10 \mathrm{mM}$ glucose (Sigma), $1 \mu \mathrm{M}$ oligomycin 533 (Agilent), and 30mM 2-deoxy-D glucose (2DG) (Sigma). Oligomycin is an inhibitor of 534 mitochondrial ATPse and 2DG inhibits the glycolytic enzyme hexokinase II. To assess mitochondrial function, cells were resuspended in $50 \mu \mathrm{L}$ Seahorse XF Base Medium (Agilent) supplemented with $2 \mathrm{mM}$ L-glutamine (Thermo Fisher), $1 \mathrm{mM}$ sodium pyruvate (Thermo Fisher), $10 \mathrm{mM}$ glucose (Sigma) and incubated for $30 \mathrm{~min}$ at $37^{\circ} \mathrm{C}$ in a $\mathrm{CO}_{2}$-free incubator. Subsequently, $130 \mu \mathrm{L}$ medium were added and cells were incubated for an additional $1 \mathrm{~h}$. Cells were sequentially treated with $1 \mu \mathrm{M}$ oligomycin (Agilent), $1 \mu \mathrm{M}$ FCCP (Agilent) and $1 \mu \mathrm{M}$ rotenone+antimycin (Agilent). OCR was measured using the Seahorse XFe96 metabolic flux analyzer (Agilent).

\section{Acknowledgments}

544 We would like to thank Dr. Lise Leclercq for critical reading of the manuscript. We thank Qusai 
546 feedback concerning conditional riboswitch. Research in Wollscheid laboratory is funded by the ETH (grant ETH-30 17-1 to B.W.) and the Swiss National Science Foundation (grant 31003A_160259 for B.W.) This project was funded by the German research foundation (DFG) through TRR130 (project2 to M.R. project 25 to J.J. and project 12 to R.E.V.). Part of this work was financially supported by the Deutsche Forschungsgemeinschaft (DFG) SFB 850 and SFB1160 Z1 to MB. Additional support was received from the German Federal Ministry of

552 Education and Research (BMBF) within the framework of the e:Med research and funding concept CoNfirm (FKZ 01ZX1708F to MB) and by MIRACUM within the Medical Informatics Funding Scheme (FKZ 01ZZ1801B to MB). Part of this work was supported by Roche, we thank Dr. Christian Klein, Roche Innovation Center Zurich for providing materials.

\section{Author contributions}

K.K. and J.J. performed experiments, and analysed data. G.A. conducted mRNA transcriptome profiling. U.S. acquired patient-data. C.B. performed western blot experiments. M.C. designed CRISPR riboswitch and generated cKO cell-lines, J.B.A. and S.N.S. performed CSC with biostatistical analysis, B.W. provided expertise in proteotype and surfaceome analysis and acquired funding. B.S. sent material for gene editing of MS4A1 gene. R.E.V. provided expertise on primary B cells and buffy coats, M.B. enabled mRNA transcriptome analysis and funding, and M.R. secured funding, developed the concept and wrote the manuscript with the help of

$$
\text { K.K., and J.J. All co-authors reviewed and edited the manuscript. }
$$

\section{Declaration of Interests}

The authors have no competing interests.

\section{References}

572 1. Pierpont TM, Limper CB, Richards KL. Past, present, and future of Rituximab-The world's first oncology monoclonal antibody therapy. Front Oncol. 2018;8(JUN). doi:10.3389/fonc.2018.00163

2. Pavlasova G, Mraz M. The regulation and function of CD20: An "enigma" of B-cell biology and targeted therapy. Haematologica. 2020;105(6):1494-1506.

578 3. MS4A1 human, P11836, https://bit.ly/2UTsTRO.

579 4. Omasits U, Ahrens CH, Müller S, Wollscheid B. Protter: interactive protein feature 
visualization and integration with experimental proteomic data. Bioinformatics. 2014;30(6):884-886. doi:10.1093/bioinformatics/btt607

5. Tedder TF, Boyd AW, Freedman AS, Nadler LM, Schlossman SF. The B cell surface molecule B1 is functionally linked with B cell activation and differentiation. $J$ Immunol. 1985;135(2):973-979. 135 (2) 973-979.

6. Kosmas C, Stamatopoulos K, Stavroyianni N, Tsavaris N, Papadaki T. Anti-CD20based therapy of B cell lymphoma: State of the art. Leukemia. 2002;16(10):2004-2015. doi:10.1038/sj.leu.2402639

7. Schioppo T, Ingegnoli F. Current perspective on rituximab in rheumatic diseases. Drug Des Devel Ther. 2017;11:2891-2904. doi:10.2147/DDDT.S139248

8. Mok CC. Current role of rituximab in systemic lupus erythematosus. Int J Rheum Dis. 2015;18(2):154-163. doi:10.1111/1756-185X.12463

9. Kläsener K, Maity PC, Hobeika E, et al. B cell activation involves nanoscale receptor reorganizations and inside-out signaling by Syk. Elife. 2014;3:e02069. doi:10.1182/BLOOD-2007-08-109769

10. He X, Kläsener K, Iype JM, et al. Continuous signaling of CD79b and CD19 is required for the fitness of Burkitt lymphoma B cells. EMBO J. 2018;37(11). doi:10.15252/embj.201797980

11. Smulski CR, Decossas M, Chekkat N, et al. Hetero-oligomerization between the TNF receptor superfamily members CD40, Fas and TRAILR2 modulate CD40 signalling. Cell Death Dis. 2017;8(2):e2601. doi:10.1038/cddis.2017.22

12. Maity PC, Yang J, Klaesener K, Reth M. The nanoscale organization of the B lymphocyte membrane. Biochim Biophys Acta. 2015;1853(4):830-840. doi:10.1016/j.bbamcr.2014.11.010

13. Vogel M, Weigand JE, Kluge B, Grez M, Suess B. A small, portable RNA device for 605 the control of exon skipping in mammalian cells. Nucleic Acids Res. 2018;46(8):e48e48. doi:10.1093/nar/gky062

14. Wollscheid B, Bausch-Fluck D, Henderson C, et al. Mass-spectrometric identification and relative quantification of N-linked cell surface glycoproteins. Nat Biotechnol. 2009;27(4):378-386. doi:10.1038/nbt.1532 
Lymphoma Cell Lines. Methods Mol Biol. 2019;1956:337-350. doi:10.1007/978-14939-9151-8_16

17. Yeung L, Hickey MJ, Wright MD. The Many and Varied Roles of Tetraspanins in Immune Cell Recruitment and Migration. Front Immunol. 2018;9:1644. doi:10.3389/fimmu.2018.01644

18. Shaffer AL, Yu X, He Y, Boldrick J, Chan EP, Staudt LM. BCL-6 represses genes that function in lymphocyte differentiation, inflammation, and cell cycle control. Immunity. 2000;13(2):199-212. doi:10.1016/s1074-7613(00)00020-0

19. Tunyaplin C, Shaffer AL, Angelin-Duclos CD, Yu X, Staudt LM, Calame KL. Direct repression of prdm1 by Bcl-6 inhibits plasmacytic differentiation. J Immunol. 2004;173(2):1158-1165. doi:10.4049/jimmunol.173.2.1158

20. Vasanwala FH, Kusam S, Toney LM, Dent AL. Repression of AP-1 function: a mechanism for the regulation of Blimp-1 expression and B lymphocyte differentiation by the B cell lymphoma-6 protooncogene. J Immunol. 2002;169(4):1922-1929. doi:10.4049/jimmunol.169.4.1922

21. Lin K-I, Tunyaplin C, Calame K. Transcriptional regulatory cascades controlling plasma cell differentiation. Immunol Rev. 2003;194:19-28. doi:10.1034/j.1600065x.2003.00040.x

22. Landau DA, Carter SL, Stojanov P, et al. Evolution and impact of subclonal mutations in chronic lymphocytic leukemia. Cell. 2013;152(4):714-726. doi:10.1016/j.cell.2013.01.019

23. D’Souza L, Bhattacharya D. Plasma cells: You are what you eat. Immunol Rev. 2019;288(1):161-177. doi:10.1111/imr.12732

24. Jellusova J. Cross-talk between signal transduction and metabolism in B cells. Immunol Lett. 2018;201(October):1-13. doi:10.1016/j.imlet.2018.11.003

25. Elhassan YS, Philp AA, Lavery GG. Targeting NAD+ in Metabolic Disease: New Insights Into an Old Molecule. J Endocr Soc. 2017;1(7):816-835. doi:10.1210/js.201700092

26. Price MJ, Patterson DG, Scharer CD, Boss JM. Progressive Upregulation of Oxidative Metabolism Facilitates Plasmablast Differentiation to a T-Independent Antigen. Cell Rep. 2018;23(11):3152-3159. doi:10.1016/j.celrep.2018.05.053

27. Garcia-Manteiga JM, Mari S, Godejohann M, et al. Metabolomics of B to plasma cell differentiation. J Proteome Res. 2011;10(9):4165-4176. doi:10.1021/pr200328f

28. Kuijpers TW, Bende RJ, Baars PA, et al. CD20 deficiency in humans results in 
648

649

650

651

652

653

654

655

656

657

658

659

660

661

662

663

664

665

666

667

668

669

670

671

672

673

674

675

676

677

678

679

680

681

impaired T cell-independent antibody responses. J Clin Invest. 2010;120(1):214-222. doi:10.1172/JCI40231

29. Uchida J, Lee Y, Hasegawa M, et al. Mouse CD20 expression and function. 2004;16(1). doi:10.1093/intimm/dxh009

30. Bunton-Stasyshyn RKA, Wells S, Teboul L. When all is not lost: considering genetic compensation in laboratory animals. Lab Anim (NY). 2019;48(10):282-284. doi:10.1038/s41684-019-0397-4

31. El-Brolosy MA, Stainier DYR. Genetic compensation: A phenomenon in search of mechanisms. PLoS Genet. 2017;13(7):e1006780. doi:10.1371/journal.pgen.1006780

32. Mahévas M, Patin P, Huetz F, et al. B cell depletion in immune thrombocytopenia reveals splenic long-lived plasma cells. J Clin Invest. 2013;123(1):432-442. doi:10.1172/JCI65689

33. Mahévas M, Michel M, Vingert B, et al. Emergence of long-lived autoreactive plasma cells in the spleen of primary warm auto-immune hemolytic anemia patients treated with rituximab. J Autoimmun. 2015;62:22-30. doi:10.1016/j.jaut.2015.05.006

34. Jellusova J, Cato MH, Apgar JR, et al. Gsk3 is a metabolic checkpoint regulator in B cells. Nat Immunol. 2017;18(3):303-312. doi:10.1038/ni.3664

35. Waters LR, Ahsan FM, Wolf DM, Shirihai O, Teitell MA. Initial B Cell Activation Induces Metabolic Reprogramming and Mitochondrial Remodeling. iScience. 2018;5:99-109. doi:10.1016/j.isci.2018.07.005

36. Cavallari M. Electroporation of human B cell lines with CRISPR reagents Delivery of ribonucleoprotein complexes using the Alt-R CRISPR-Cas9 System and the Neon ${ }^{\circledR}$ Transfection System into Ramos, BJAB, and DG75 cells. :1-6. www.idtdna.com.

37. Ahlen MT, Husebekk A, Killie MK, Skogen B, Stuge TB. T-cell responses associated with neonatal alloimmune thrombocytopenia: isolation of HPA-1a-specific, HLADRB3*0101-restricted CD4+ T cells. Blood. 2009;113(16):3838-3844. doi:10.1182/blood-2008-09-178475

38. Almén MS, Nordström KJV, Fredriksson R, Schiöth HB. Mapping the human membrane proteome: A majority of the human membrane proteins can be classified according to function and evolutionary origin. BMC Biol. 2009;7:50. doi:10.1186/1741-7007-7-50

39. Kläsener K, Yang J, Reth M. Study B Cell Antigen Receptor Nano-Scale Organization by in Situ Fab Proximity Ligation Assay. Vol 1707.; 2018. doi:10.1007/978-1-49397474-0_12 
682 40. Müller S, Rycak L, Afonso-Grunz F, et al. APADB: a database for alternative polyadenylation and microRNA regulation events. Database (Oxford). 2014;2014.

684 doi:10.1093/database/bau076

685

41. Ritchie ME, Phipson B, Wu D, et al. limma powers differential expression analyses for

686 RNA-sequencing and microarray studies. Nucleic Acids Res. 2015;43(7):e47.

687 doi:10.1093/nar/gkv007

688

42. Sergushichev AA. An algorithm for fast preranked gene set enrichment analysis using

689 cumulative statistic calculation. bioRxiv. 2016:060012. doi:10.1101/060012

690 
Figure Legends:

692

\section{CD20 expression and localization on WT Ramos B cells}

a Flow cytometry analysis showing the expression of IgM-BCR, IgD-BCR, CD19 and CD20 on Ramos WT cells compared to unstained control (CTRL), n=18 b Fab-PLA of the proximity of CD20 to IgD-BCR or CD20 to IgM-BCR on resting (R), or 5 min pervanadate activated (A) WT Ramos B cells. Representative microscope images (left) of PLA-signals shown in red and nuclei in blue. Scale bar $10 \mu \mathrm{M}$. Scatter dot plot represent the mean (red bar) and SD of PLA signals (Signal Counts), $\mathrm{n}=3 \mathbf{c}$ Schematic drawing of the proposed model of IgM and IgD-BCR protein islands with coreceptors on the surface of human resting B cells.

\section{Loss of CD20 results in altered expression of $B$ cell surface markers and transient} activation

a Timeline of developmental states in CD20KO Ramos cell-line generation. MS1A4 gene targeting with CRISPR/Cas9 at day 0, developmental stage at day 3-6 shown as CD20KO-new (KO-N, orange), at day 10-17 shown as CD20KO-intermediates (KO-I, light blue), after 1 month shown as CD20KO-late (KO-L, dark blue). b Flow cytometry analysis showing overtime expression of surface molecules of KO-N, KO-I, and KO-L Ramos cells compared to WT and unstained control (grey), n=18. c Representative western blot analysis of KO-L Ramos B celllysates (right) compared to WT (left). Lysates were taken 20 days after MS4A1 gene targeting, $\mathrm{n}=14 \mathbf{d}$ Fab-PLA analysis of IgM-BCR proximity to CD19 in resting WT Ramos B cells compared to the unstimulated KO-N cells 3 days after MS4A1 gene targeting or with empty vector (not shown), Scale bar $10 \mu \mathrm{M}$ (left). PLA microscope images were quantified as scatter dot plot with mean and SD (right), $n=3$ e Intracellular flow cytometry analysis of phosphorylated Akt (pAkt-Ser473) or phosphorylated Syk (pSyk-Tyr525,526) of KO-N cells compared to WT. Gating used to analyse high IgM-BCR expressing KO-N cell population for pSyk or pAkt levels respectively. f Flow cytometry analysis showing the overtime expression of the B cell-specific surface activation markers CD86, and CD69 of KO-N compared to WT.

\section{In vitro and in vivo treatment with rituximab (RTX) leads to CD20KO phenotype}

a Flow cytometry analysis after treatment with RTX for 3 days showing the loss of CD19, IgMcontrol (Ctrl), n=3 b Flow cytometry analysis of negatively selected naïve B cells from peripheral blood of a healthy donor were treated with RTX for 60min (HD-RTX) and compared 
to untreated (HD), to plasma cells (PC) of the same donor or left untreated and unstained (Ctrl) $\mathrm{n}=3$. $\mathbf{c}$ Example of $\mathrm{B}$ cells taken from EDTA whole blood samples of RA patient undergoing RTX treatment after $0,15,30,60,120 \mathrm{~min}$. RTX [1mg/mL] flow rate $50 \mathrm{~mL} / \mathrm{h}$. Flow cytometry staining of CD19+/ CD27-/ IgM+ selected B cells shows internalization of IgM and CD19, n=2.

\section{Increased Plasma cell differentiation in CD20KO Ramos B cells}

a Flow cytometry analysis showing size (FSC-A) and expression of plasma cell markers TACI, CD138, and AID on KO-N, KO-I, and KO-L Ramos B cells compared to WT and unstained control (Ctrl), n=6. b Expression of surface IgA-BCR (left) and ELISA of IgA secretion (right) of KO-L compared to Ramos WT, PBS and RPMI control. c Summarized intracellular flow cytometry analysis of 6 independently generated CD20KO Ramos B cell-lines showing the fold change (FC) of Pax5 and Blimp1 expression of KO-L Ramos B cell lines compared to WT. d Representative examples of western blot analysis for B cell differentiation markers of KO-L Ramos B cells compared to WT. Lysates were taken 20 days after induction of CD20KO. $n=3$. e PLASMA UP GENES Heatmap, expression of plasma cell differentiation up-regulated genes. Color code indicates the row-wise scaled intensity across the samples. Genes are ranked according to their Log2 fold FC in WT (left) vs. KO-L (right) $\mathrm{n} \geq 3$. f Example of enrichment plot (Curve) of plasma cell differentiation up-regulated genes in one patient. g Enrichment barcode illustrating the distribution of plasma cell differentiation up-regulated genes (colored segments) in every individual patient. from GSE37168. Treated patients were ordered from left to right based on their enrichment score, from high to low. Significant enrichment scores are depicted by “*”.

\section{Metabolic switch of CD20KO Ramos B cells}

a Oxygen consumption rate (OCR) of KO-L compared to WT Ramos cells. Metabolite flux analysis was performed in triplicates and is displayed as mean \pm SD. One, out of 3 experiments, is shown. Used inhibitors: $\mathrm{A}=$ Oligomycin, $\mathrm{B}=\mathrm{FCCP}, \mathrm{C}=$ Rotenone + Antimycin. $\mathbf{b}$

752 Mitochondrial mass staining with Mito Tracker Red-CMXRos in KO-L compared to WT

753 Ramos cells and normalized to volume of WT Ramos cells. $\mathrm{n}=4$. c NAD ${ }^{+}$levels of KO-L compared to WT Ramos cells determined with untargeted metabolomic profiling, $n=3$. d Mitochondrial superoxide levels stained with MitoSox Red. KO-L cells, WT Ramos cells and unstained control (Ctrl) are shown, $\mathrm{n}=4$. e Survival rates (propidium iodide negative cells) of KO-L and WT Ramos cells after Buthionine sulfoximine (BSO) titration. The experiment was performed three times and mean MFI values were normalized to day 0 . f Extracellular 
acidification rate (ECAR) of KO-L and WT Ramos cells as a measure of glycolysis is shown. The measurement was performed in technical triplicates and is displayed as mean $\pm \mathrm{SD}$. One, out of 3 independent experiments, is shown $\mathrm{D}=$ Glucose, $\mathrm{A}=$ Oligomycin, $\mathrm{E}=2 \mathrm{DG} . \mathbf{g}$ Representative western blot analysis of Hexokinase II (HK II) protein levels of KO-L and WT Ramos cells. Lysates were taken 20 days after induction of CD20 KO and data are representative for at least three independently generated CD20 KO Ramos B cell lines. $\mathbf{h}$ Levels of the glycolytic intermediates glucose 6-phosphate (G6P), fructose 6-phosphate (F1,6BP), and pyruvate as determined by untargeted metabolomic profiling are shown, $n=3$. i Cell death as determined by propidium iodide (PI) staining after treatment with BPTES, $n=3$. $\mathbf{k}$ Proliferation index determined with MFI of CellTrace staining per day (d) of KO-L cells cultivated in galactose compared to WT Ramos cells, n=3. I Levels of UDP-galactose, galactose-1-phosphate (G 1-P) and galactonate as determined by untargeted metabolomic, $\mathrm{n}=4$.

\section{Extended Figure 1:}

\section{CRISPR/Cas9 CD20KO generation}

Schematic of CRISPR-mediated gene targeting site in exon 3 of the scaled exon-intron organization of the human MS4A1 gene. Sequence of CRISPR- single guide RNA (sgRNA) as depicted.

\section{Extended Figure 2:}

The IgM-BCR and CD19 are indispensable for CD20KO B cell activation

Flow cytometry analysis showing surface molecule expression on a CD19/20DKO-L, CD19KO, and CD20KO-L Ramos cells compared to WT and unstained control (grey) or $\mathbf{b}$ on grey).

\section{Extended Figure 3:}

Proof of the Principle: conditional CD20KO (cKO)

a Schematic of CRISPR-mediated insertion of an aptamer-controlled exon (c-exon) in intron 34 in the human MS4A1 gene generating a CD20 conditional KO (cKO-top). Tetracyclin (Tet) induces c-exon skipping and restores the ORF of the MS4A1 gene (cKO-Tet, bottom). b Induction of cKO Ramos B cells with $6 \mu \mathrm{M}$ Tet for $12 \mathrm{~h} 30$ days after transfection restored the WT phenotype. Examples of flow cytometry analysis of surface receptors CD20, CD19, IgM, 
CD22, CD81, and CD40 of cKO-Tet (red) compared to cKO Ramos cells (blue), WT and Tetinduced WT Ramos cells (Ramos-Tet) as control.

\section{Extended Figure 4}

\section{RTX treatment activates primary naïve B cells via the canonical BCR pathway}

a PLA on resting (UT), 15 min RTX treated (RTX) or 5 min pervanadate activated (A) negatively selected naïve B cells from the peripheral blood of a healthy donor. Scatter dot plots showing the mean and SD of quantified results of the proximity of CD19 to IgM-BCR, Ig $\alpha$ to

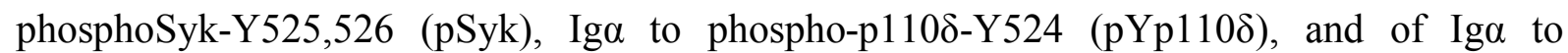
phosphoAkt-Ser473 (pAkt), n=4.

\section{Extended Figure 5}

\section{Surfaceome analysis of CD20KO-N and KO-L}

a Volcano plot showing the $\log 2 \mathrm{FC}$ of $\mathrm{KO}-\mathrm{N}$ cell surface proteins compared to Ramos WT cells 10 days after gene targeting. b Top50 of significant (p-value $<0.05$ ) of up (right) or downregulated (left) KO-N cell surface proteins compared to WT Ramos cells. c Volcano plot showing the Log2 FC of KO-L cell surface proteins compared to Ramos WT cells 1 month after gene targeting. d Top50 of significant (p-value $<0.05$ ) of up (right) or downregulated (left) KOL cell surface proteins compared to WT Ramos cells. e Voronoi tree map of KO-N surfaceome (left) or KO-L (right). All quantified proteins were mapped and hierarchically grouped according to their functional classification. For representation the $\log 2 \mathrm{FC}$ values have been scaled from $0-1$. The colors represent the Log2 FC between the compared conditions.

\section{Extended Figure 6}

\section{Expression of Pax5 and Blimp1 remain unchanged in CD19/20DKO and BCR/20DKO}

\section{Ramos cells}

a Representative examples of western blot analysis for B cell differentiation markers Pax5 and Blimp1 of CD19KO, CD19/20DKO-L, and KO-L Ramos B cells compared to WT. b Western blot analysis showing B cell differentiation markers Pax5 and Blimp1 of BCRKO, BCR/20DKO-L, and KO-L Ramos B cells compared to WT. All lysates were taken 20 days after induction of CD20 KO. 


\section{Figure 1}

CD20 expression and localisation on WT Ramos B cells

a

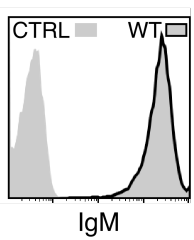

b

$\lg \mathrm{D}: \mathrm{CD} 20$

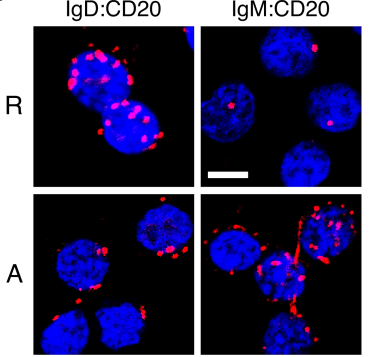

C

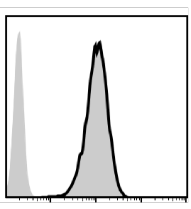

CD19

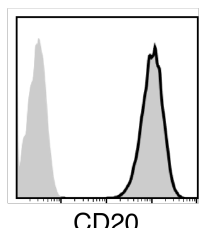

CD20

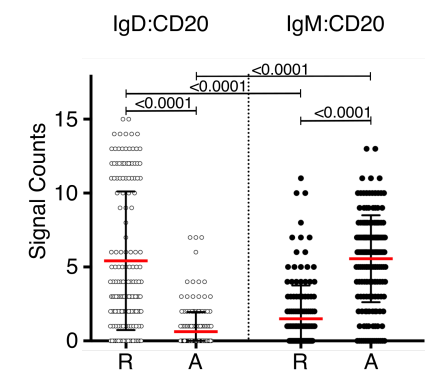

C IgM protein island

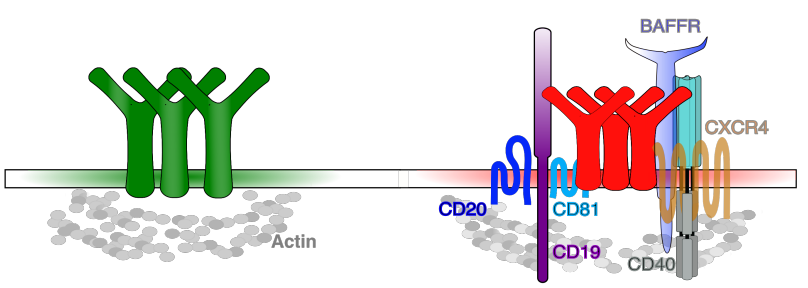

Figure 2

Loss of CD20 results in altered expression of $B$ cell surface markers

a

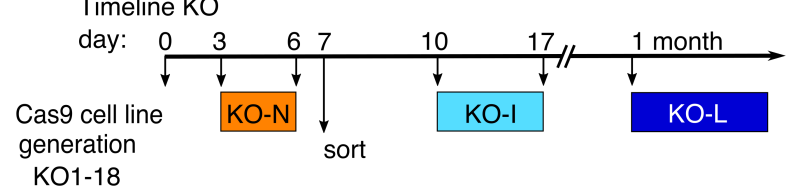

b
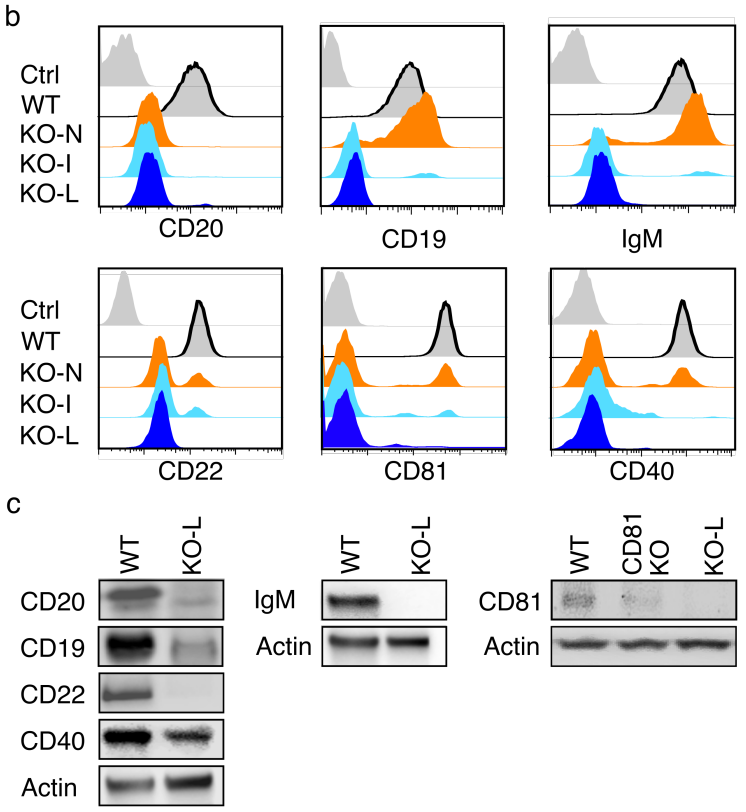

CD19
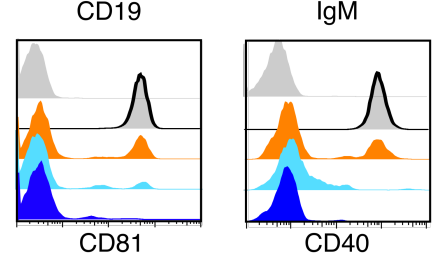

.
IgD protein island e
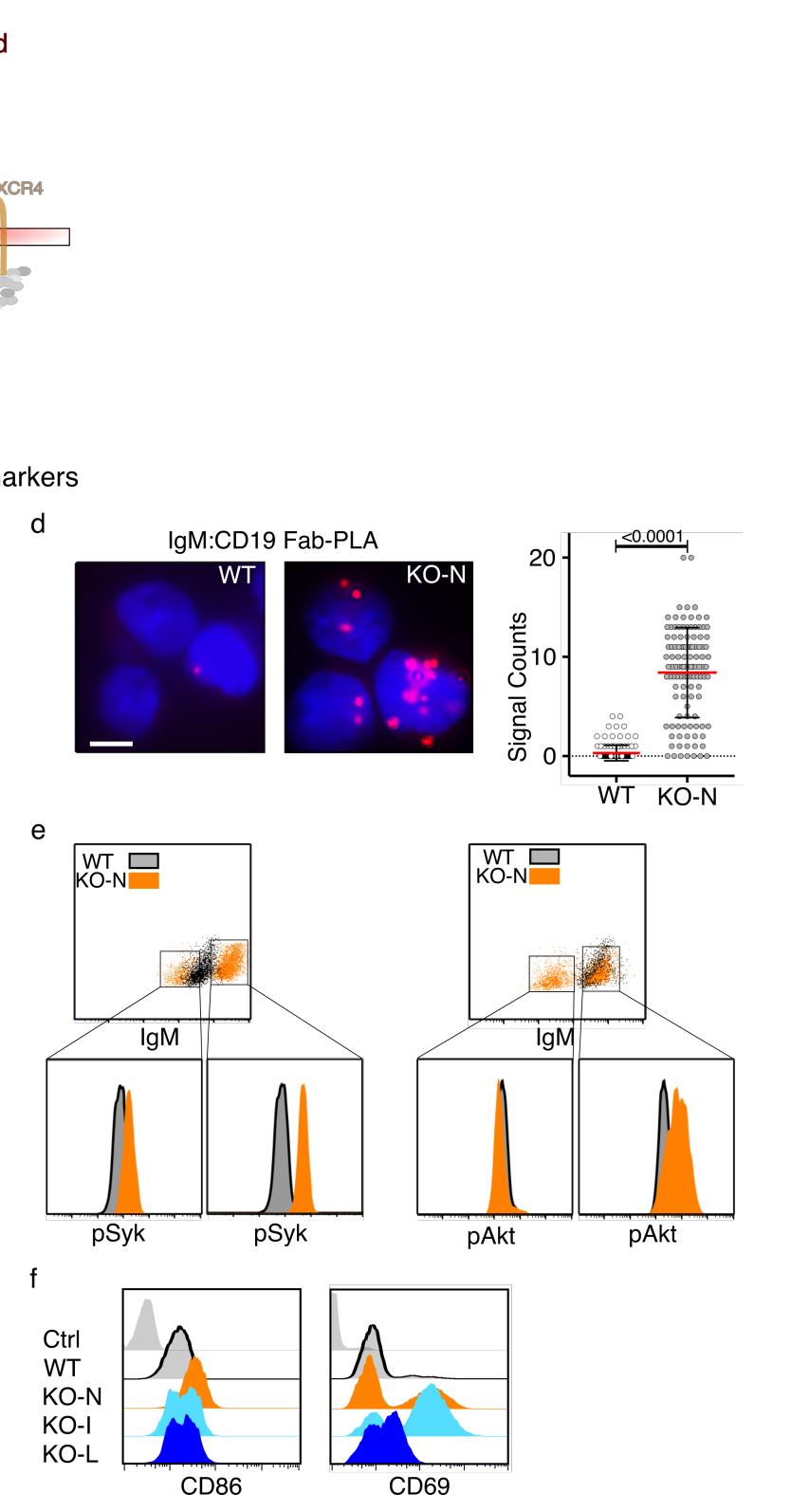
Figure 3

In vitro and in vivo treatment with RTX leads to CD20KO phenotype

a
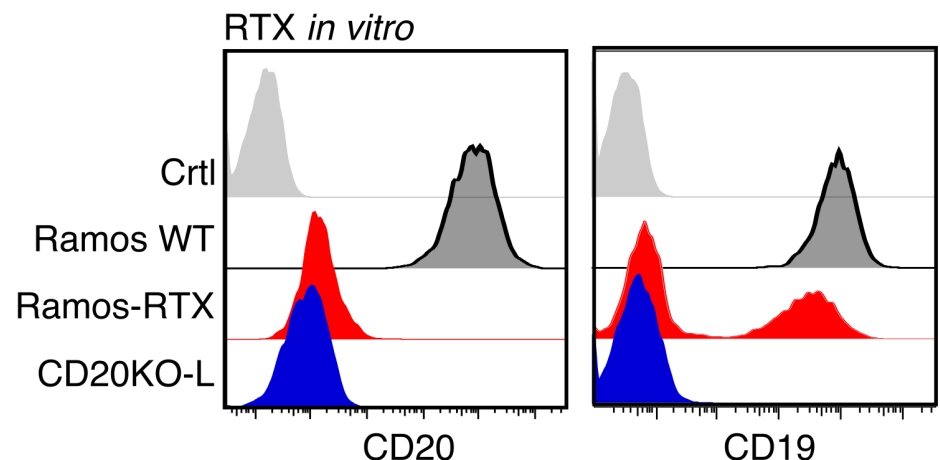

CD19

b
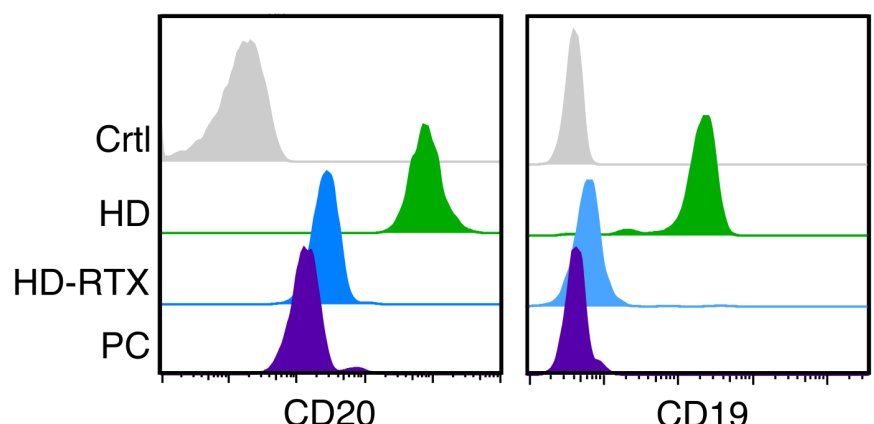

CD19

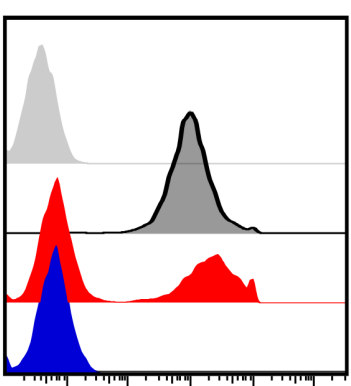

$\lg \mathrm{M}$

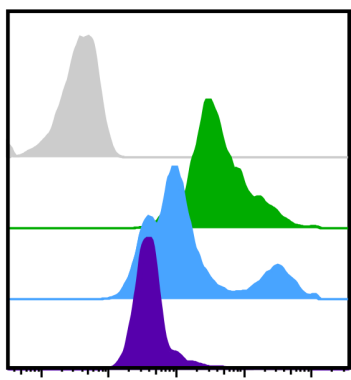

$\lg M$

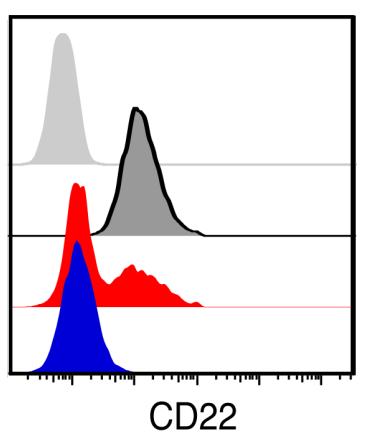

CD22

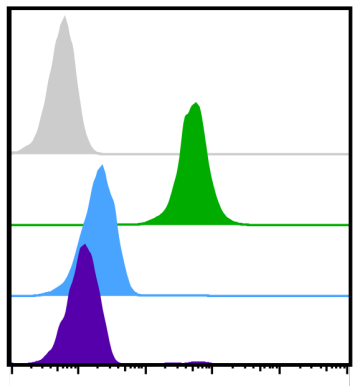

CD22

C
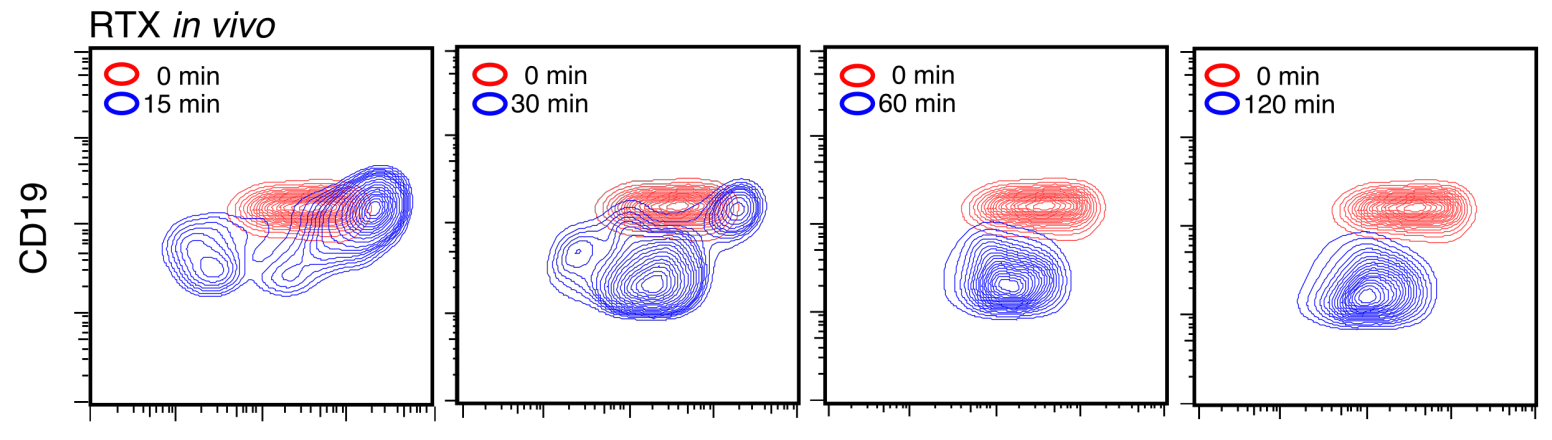

$\lg \mathrm{M}$ 


\section{Figure 4}

Increased Plasma cell differentiation in CD20KO Ramos and CLL B cells

a

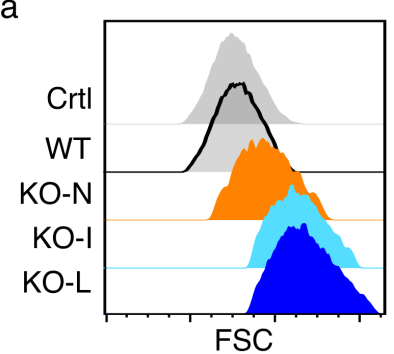

b

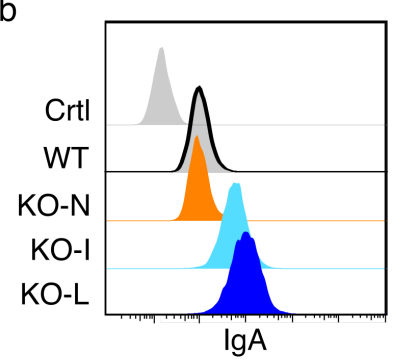

e

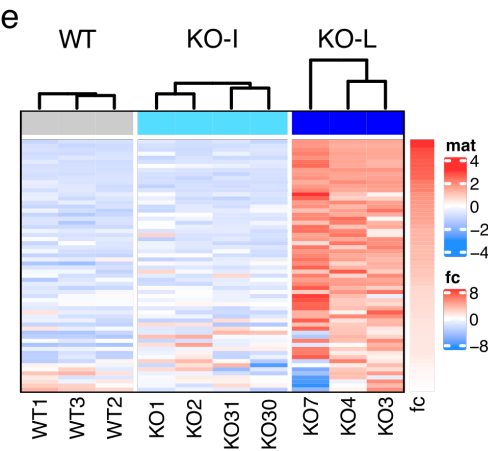

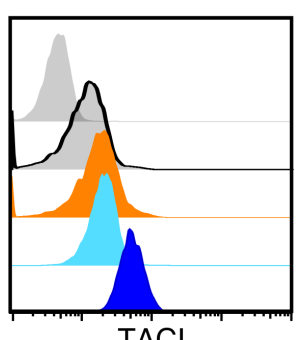

TACI

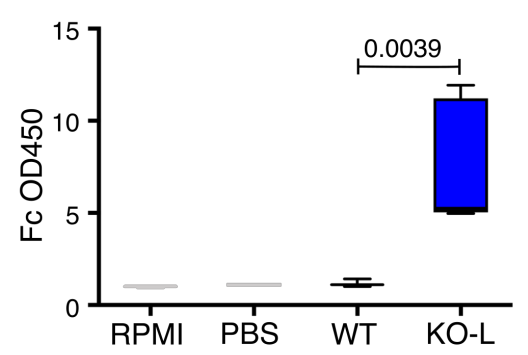

f

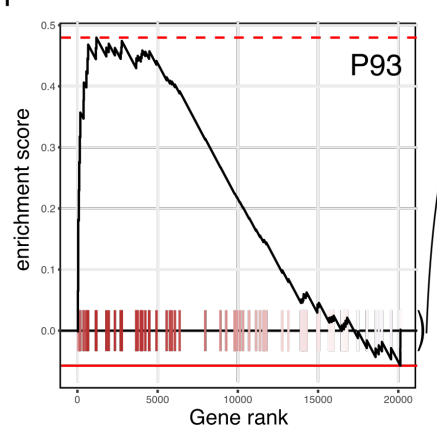

d

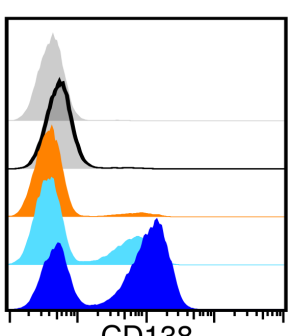

$\mathrm{CD} 138$

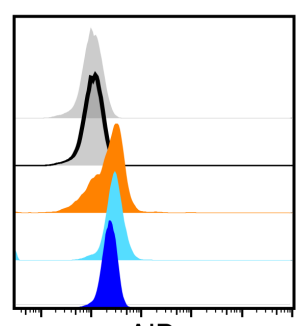

AID
C Pax5 norm. Blimp1 norm.

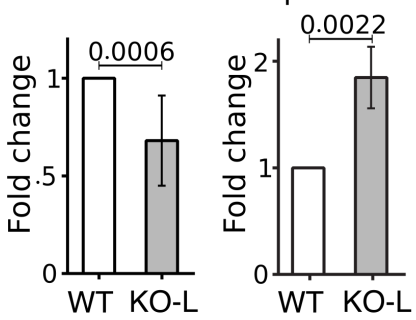

g

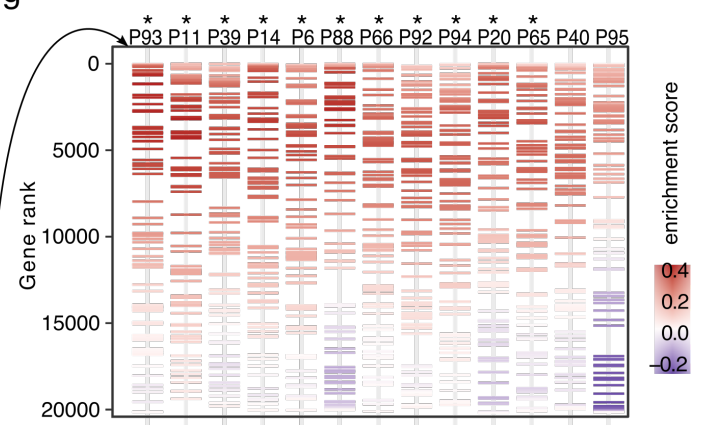


Figure 5

Metabolic switch of CD20KO Ramos B cells
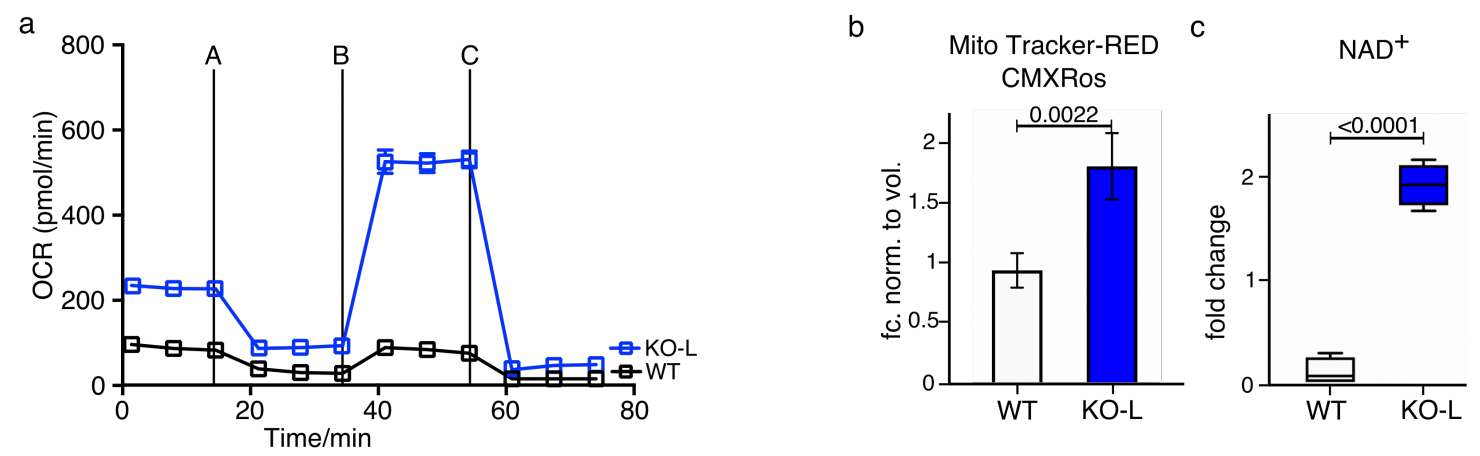

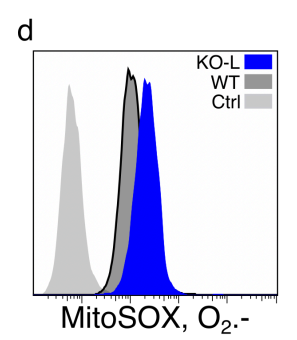

$g \quad$ WT KO-L
HK II $~$
Actin $~$

$\mathrm{h}$
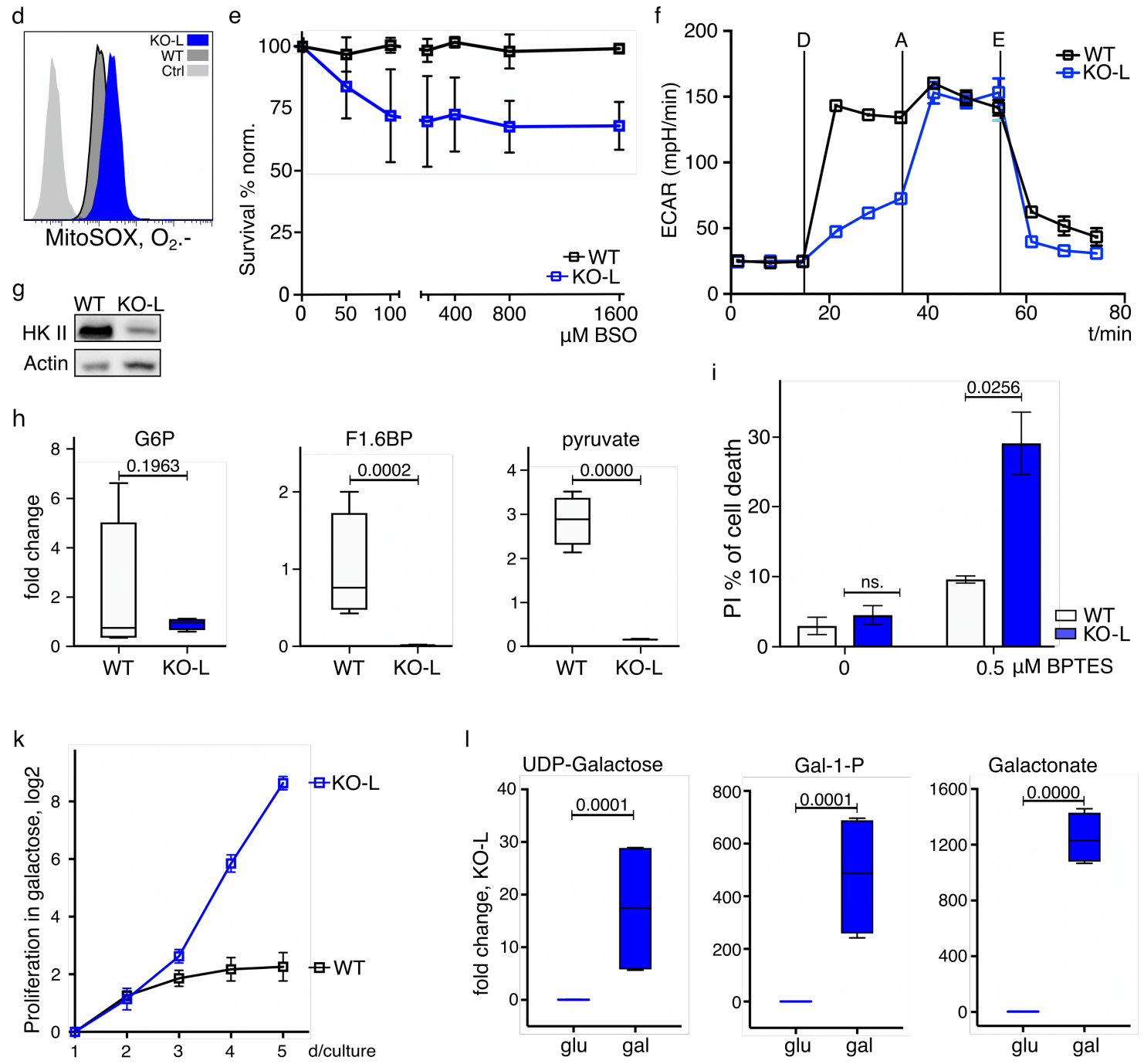
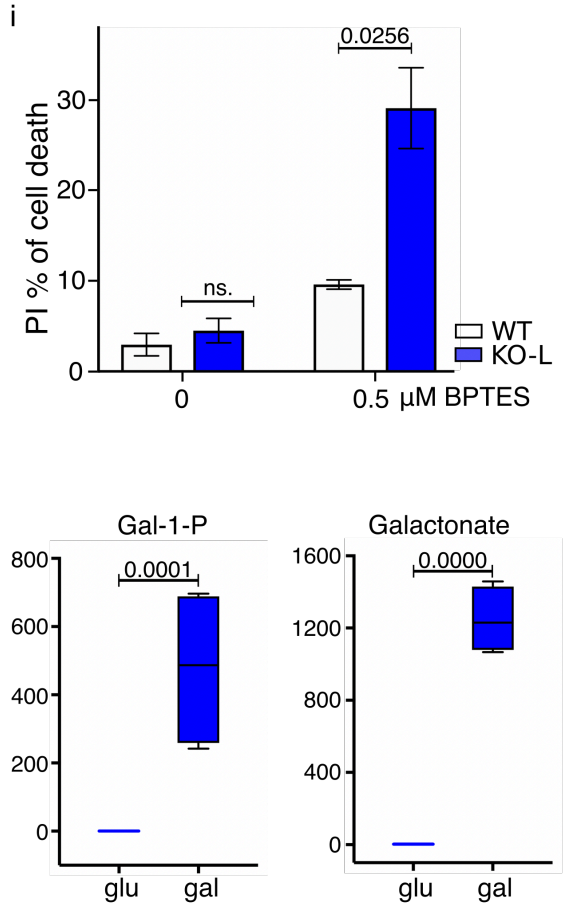
Extended Figure 1

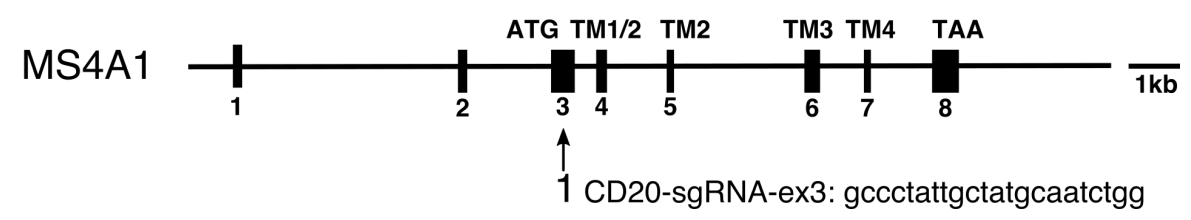

Extended Figure 2:

The BCR and CD19 are indispensable for CD20KO B cell activation

a

b
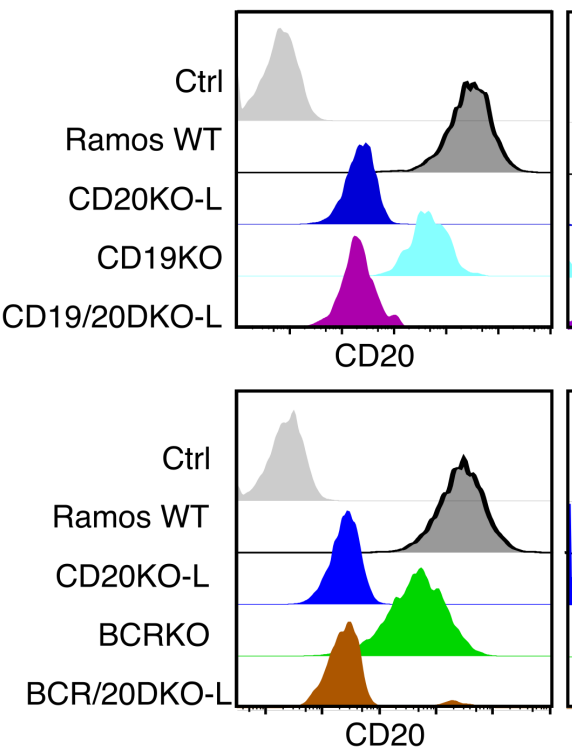

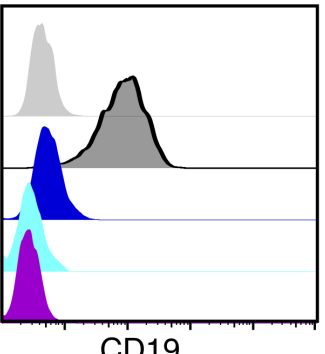

CD19

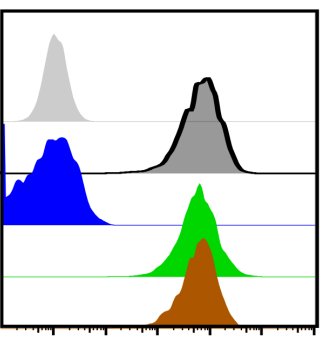

CD19

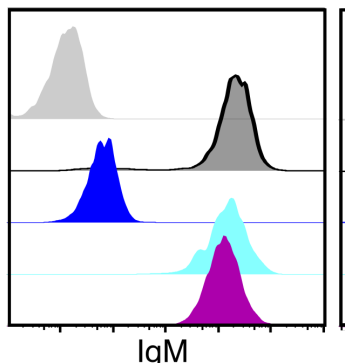

$\lg M$

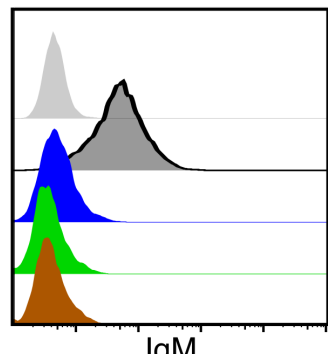

$\lg M$

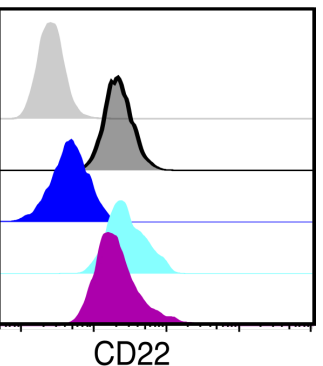

CD22

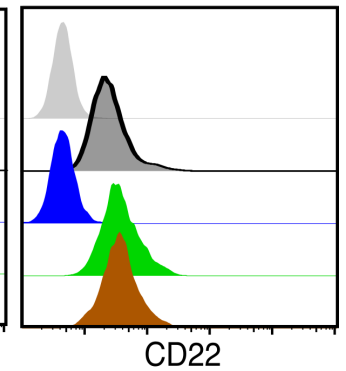




\section{Extended Figure 3}

Proof of Principle: conditional CD20KO

a

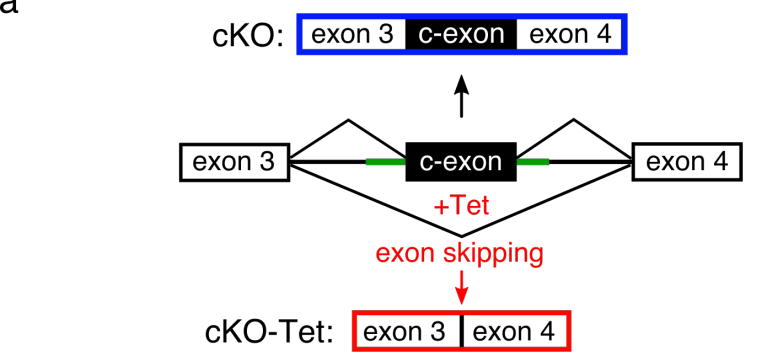

b
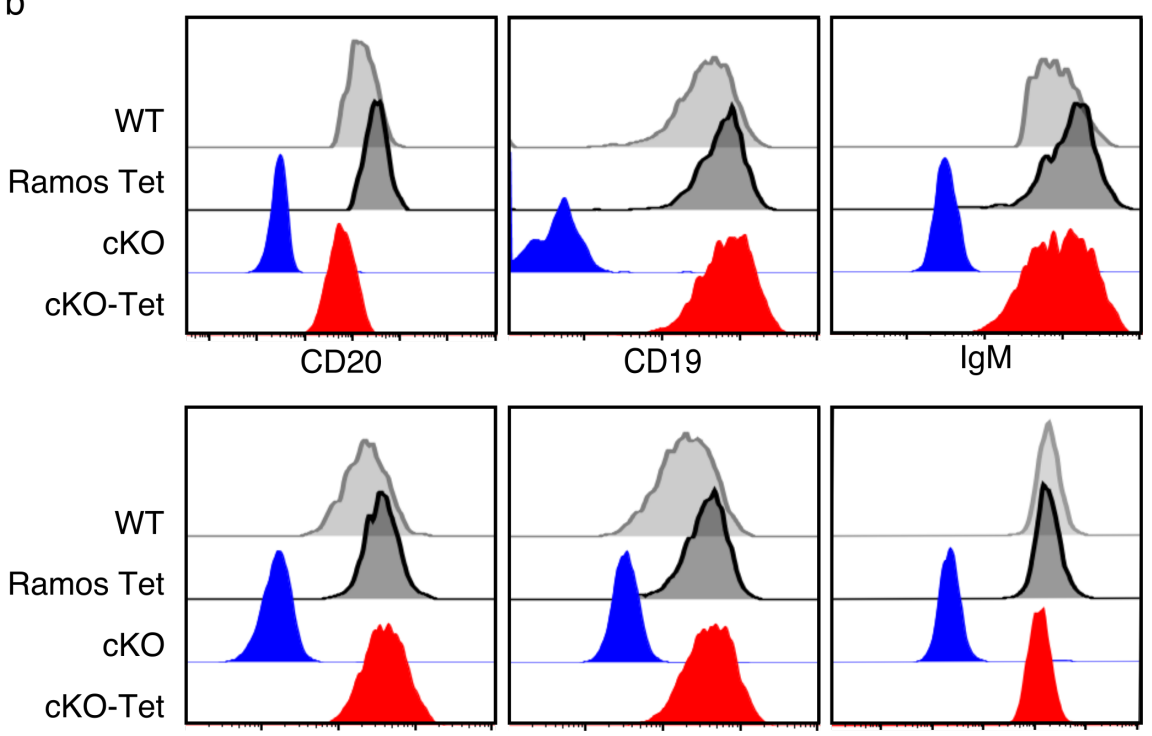

\section{Extended Figure 4}

a

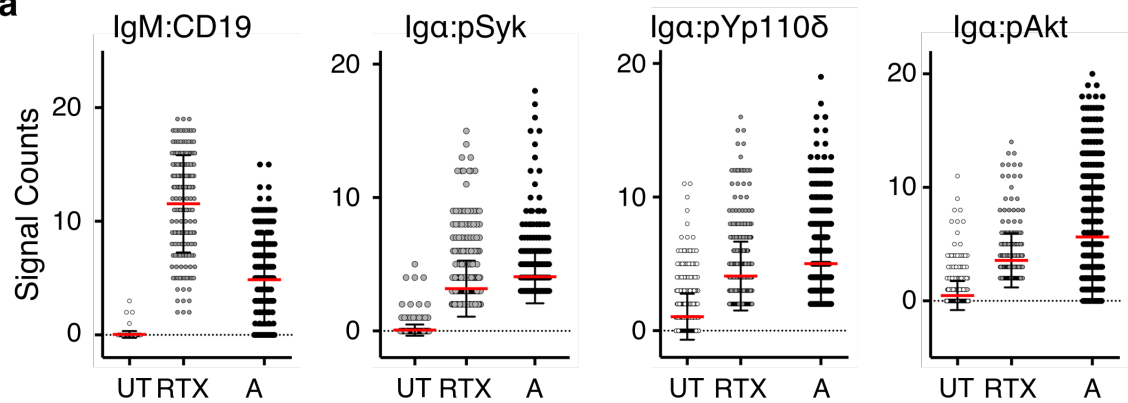

b

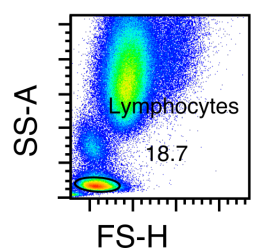

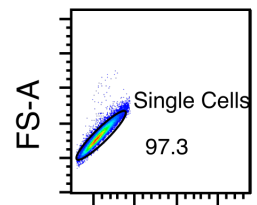

FS-H

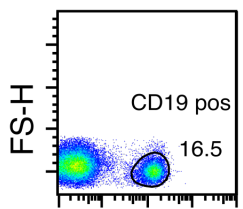

CD19

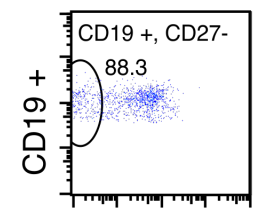

CD27 
Extended Figure 5

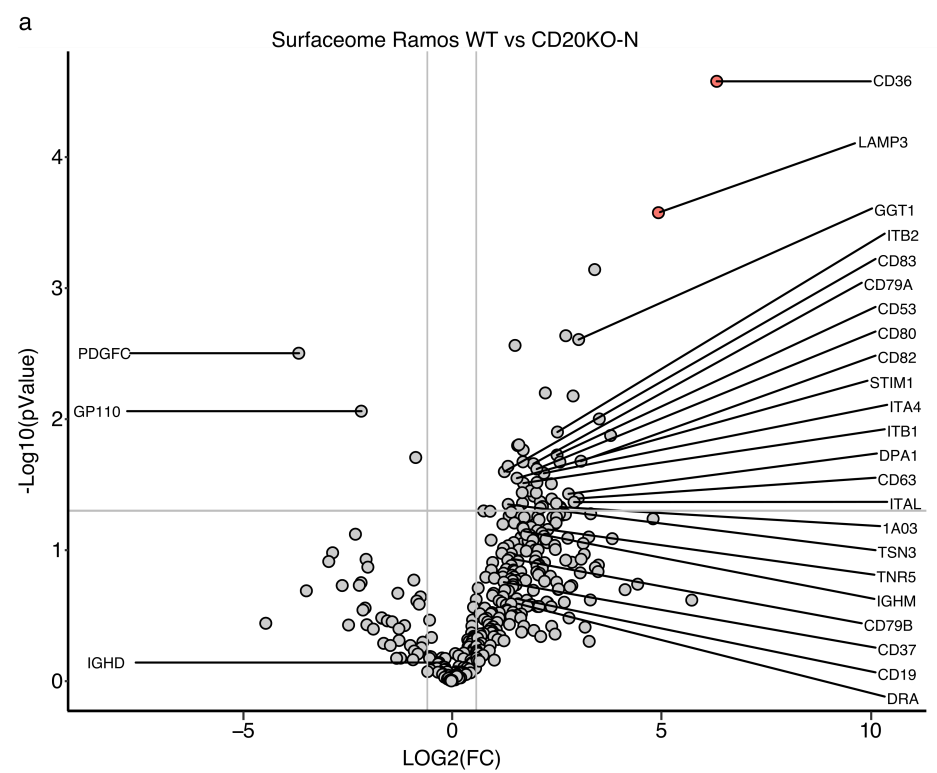

C

Surfaceome Ramos WT vs CD20KO-L

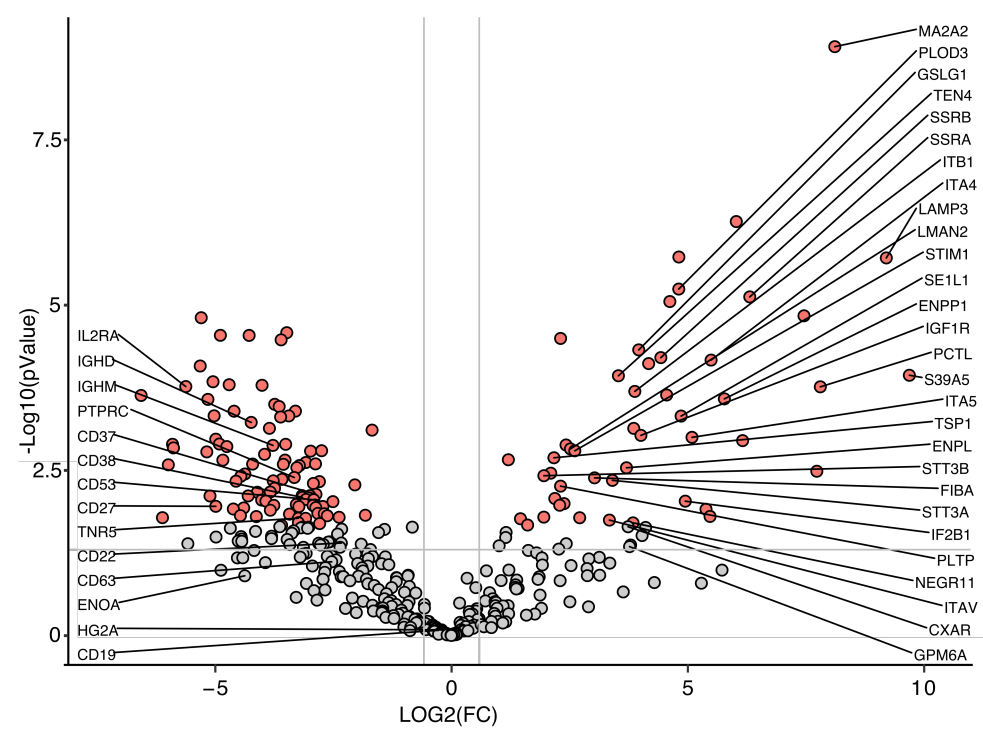

e

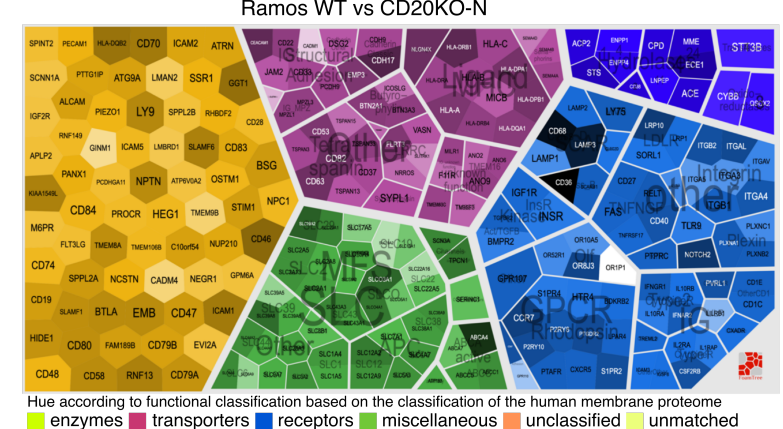

b
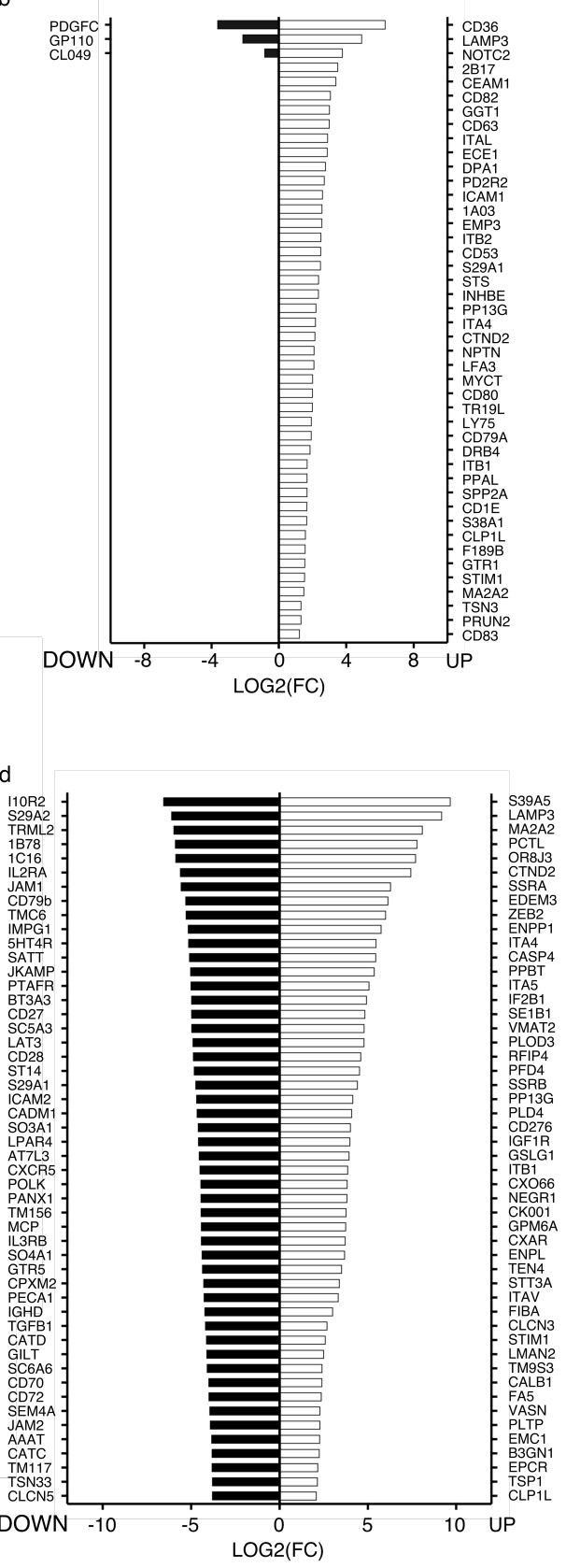

Ramos WT vs CD20KO-L

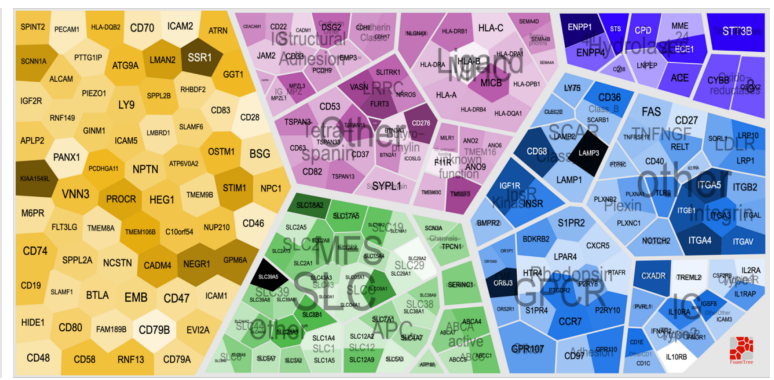




\section{Extended Figure 6}

a

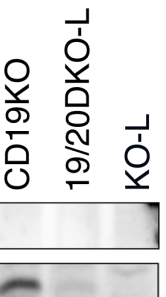

b

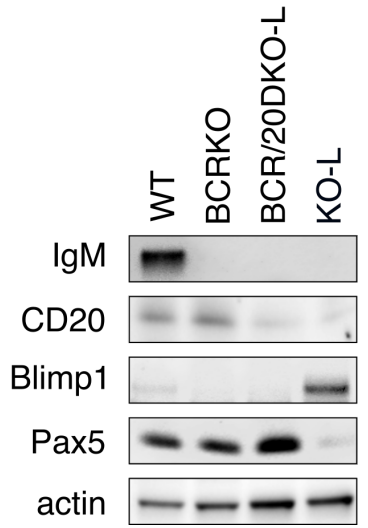

